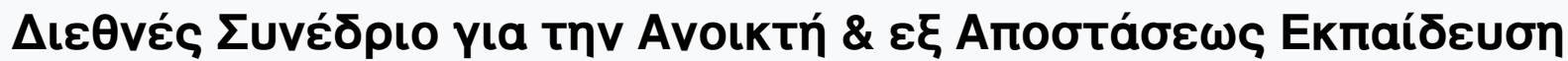

Tóp. 8, Ap. 2A (2015)

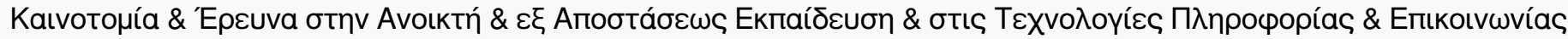

| ПраKüiká

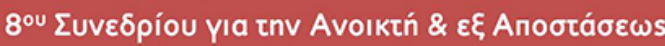

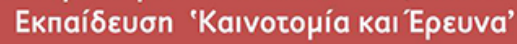

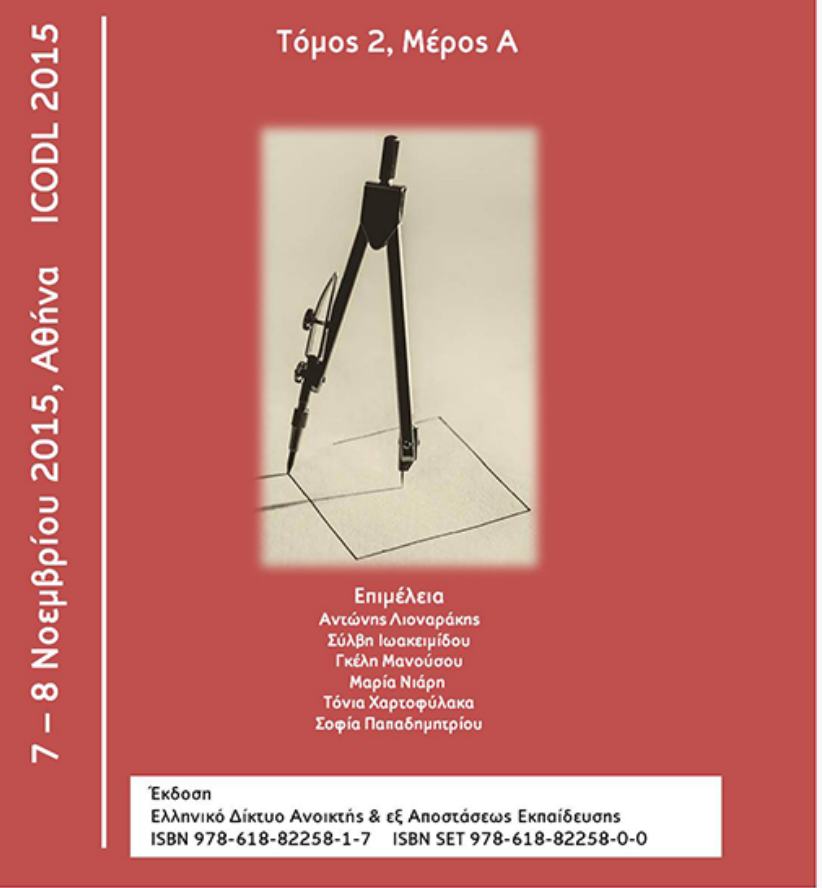

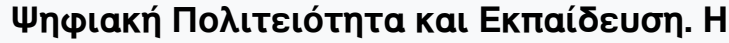

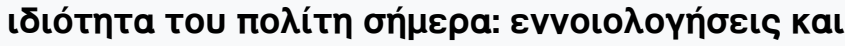

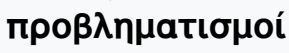

\section{Mapía Mapıvákn}

doi: $10.12681 /$ icodl. 47 


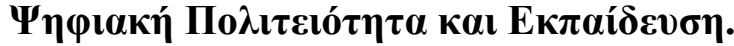

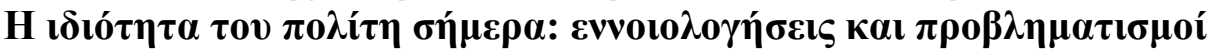

\section{Digital Citizenship and Education: conceptualizing and examining the idea of today's digital citizen and its formulation through the educational process}

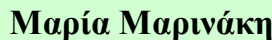

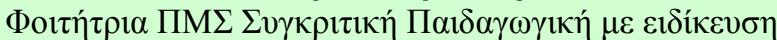

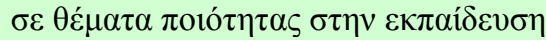 \\ ЕКПА \\ marinaki.maria@gmail.com
}

\begin{abstract}
Globalization and modern technologies dominate in the contemporary discourse on the history of modern man. In view of the fact that they constitute the main axes of today's analyses, they seem to have established the most comprehensive and predominant twofold, which is strongly and profoundly influencing the capacity of the individual as an active, consistent and responsible subject. Today's (active) 'world citizen' or 'global citizen' seems to be pertinent to a transcending form of the State which expands and diffuses in space and time, unveiling the dynamic and multifaceted context that defines the lives of the individuals. Being a citizen bears by definition the moral and factual obligation to respond rationally, respectfully, sensibly and responsibly to the demands of a highly heterogeneous environment that appreciates and values diversity and complexity. At the same time, as a social being, today's global citizen is expected to contribute to a harmonious 'symbiosis' conforming to the societal norms of the actual sphere of human coexistence but also within the boundless spectrum proposed by cyberspace in parallel and alternatively to the idea of the modern State. The new type of (digital) citizenship sets new requirements and requisites in accordance with those of the digital era, creating a sophisticated frame in which it envisages today's global citizen. In its turn, education reveals itself as the most appropriate means of strengthening and supporting the identity of the global citizen through the complex and sometimes misleading paths evoked by the contemporary technological progress and development, in an attempt to diminish the profusion of the deceptive lures of technology and avoid the call of the Sirens in today's globalized world.
\end{abstract}

Key-words: digital citizenship, citizen, ICT, rights, skills, education, knowledge

\section{Пврі́ $\eta \Psi \psi \eta$}

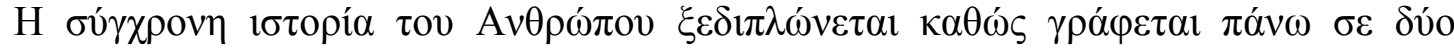

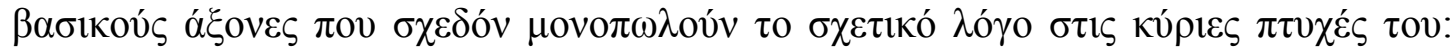

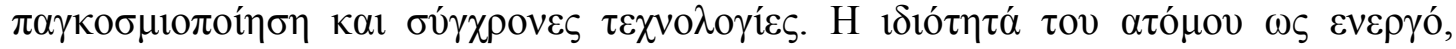

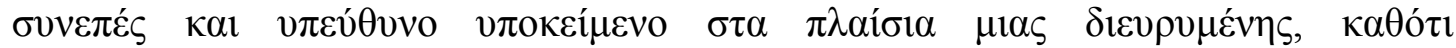

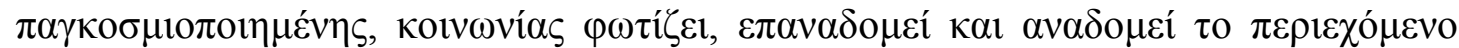

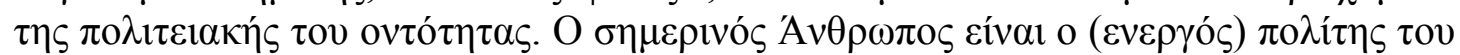

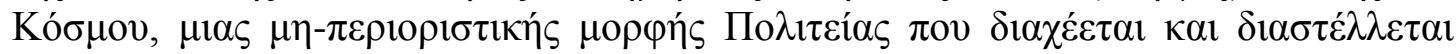




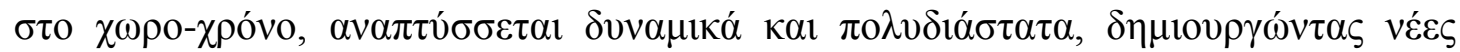

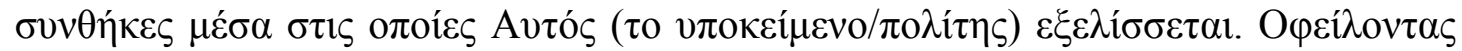

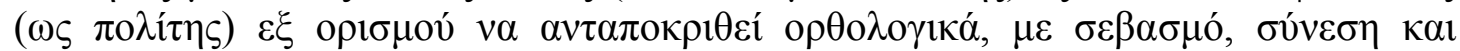

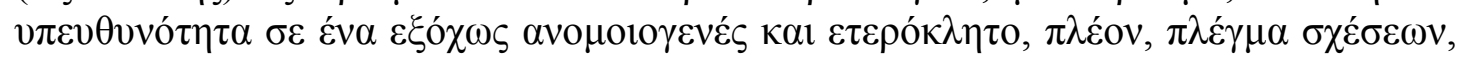

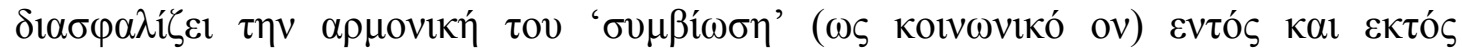

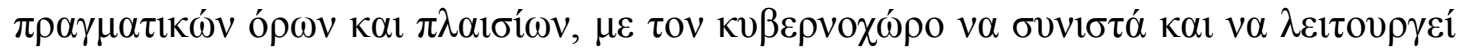

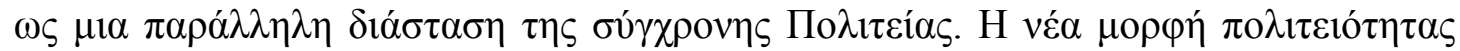

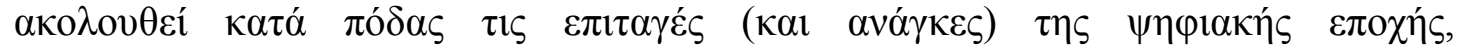

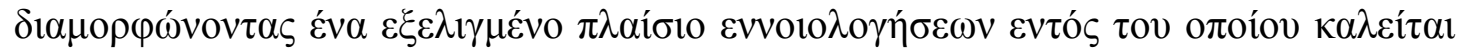

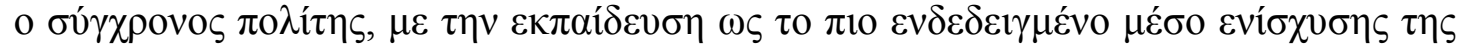

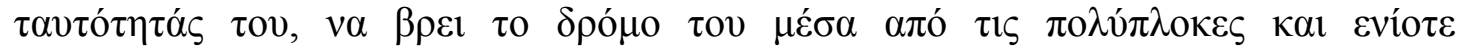

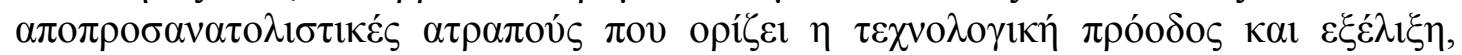

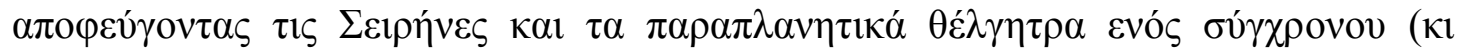

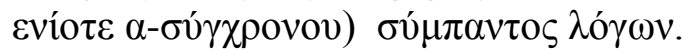

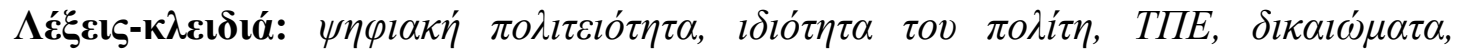
$\delta \varepsilon \xi \xi o ́ \tau \eta \tau \varepsilon \varsigma, \varepsilon \kappa \pi \alpha i \delta \varepsilon v \sigma \eta, \gamma v \omega ́ \sigma \eta$

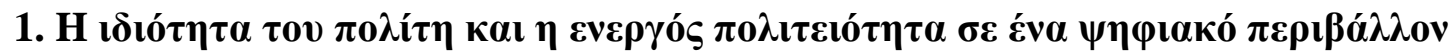

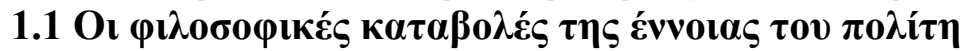

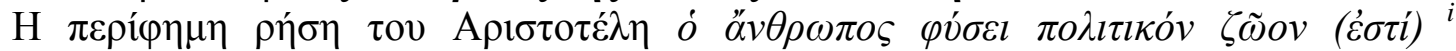

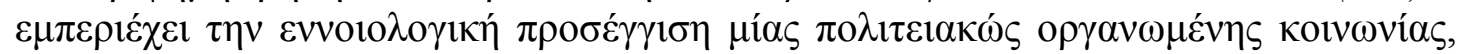

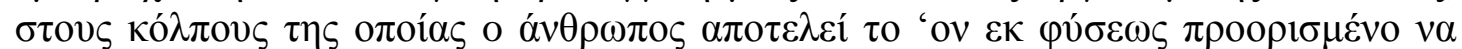

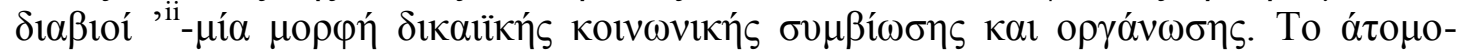

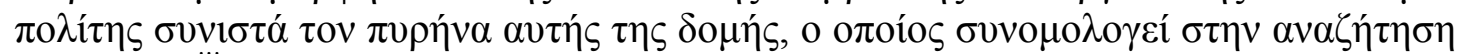

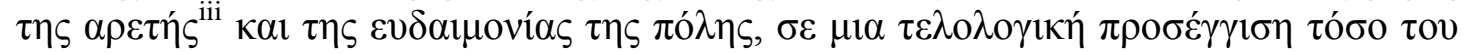

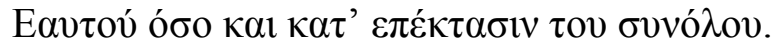

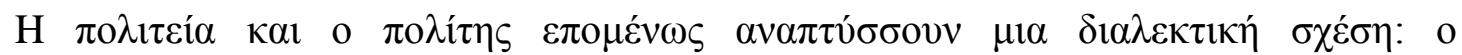

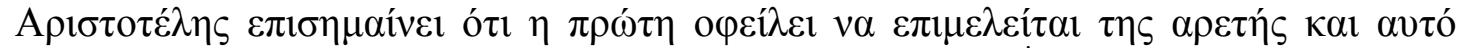

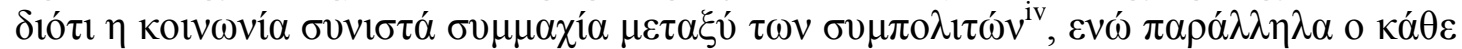

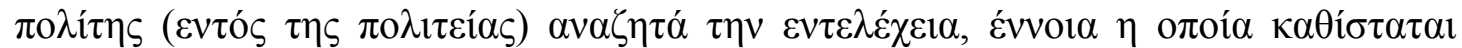

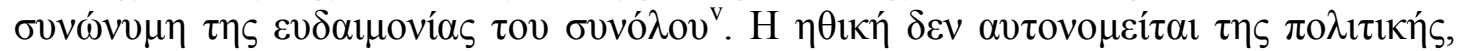

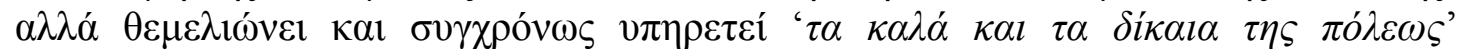

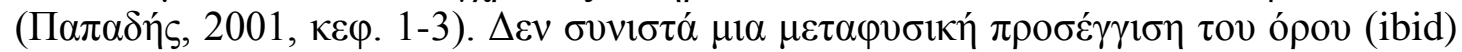

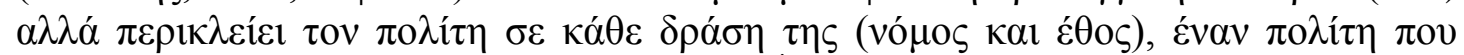
$\gamma v \omega \rho i \zeta \varepsilon l \kappa \alpha l \delta \dot{v} v \alpha \tau \alpha l v \alpha \alpha ́ \rho \chi \varepsilon l \kappa \alpha l v \alpha \alpha \dot{\alpha} \rho \varepsilon \tau \alpha \mathrm{l}^{\mathrm{vi}}$.

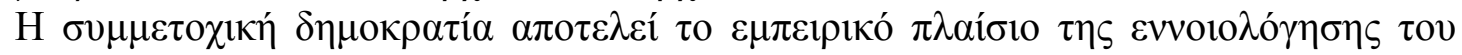
A

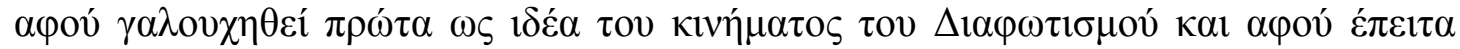

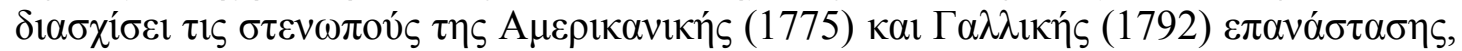

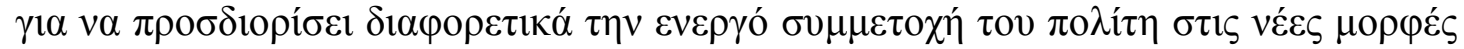

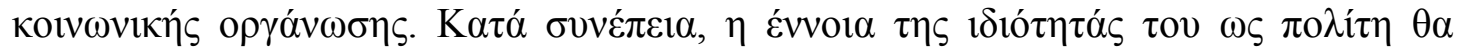

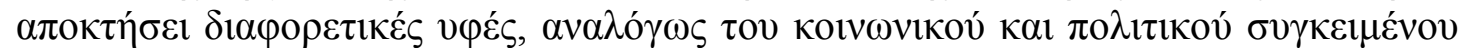

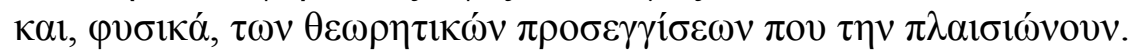

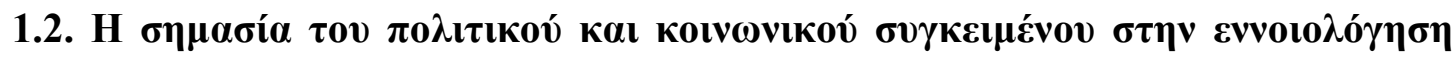 $\tau \eta \varsigma \pi 0 \lambda ı \tau \varepsilon เ o ́ \tau \eta \tau \alpha \varsigma$}

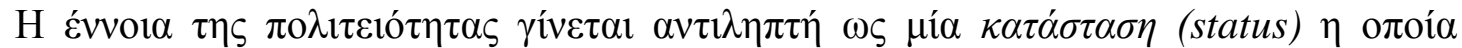

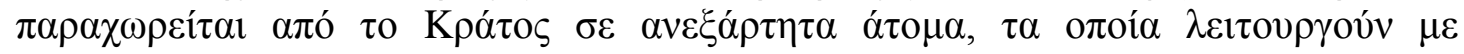




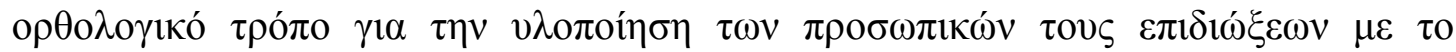

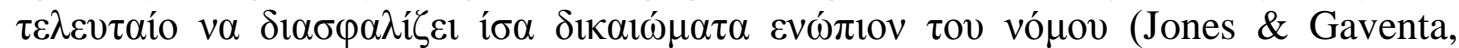

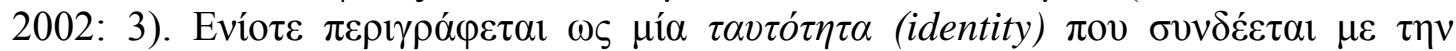

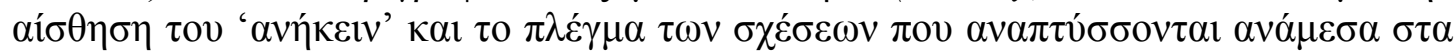

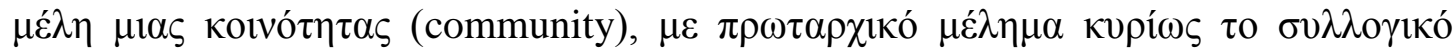

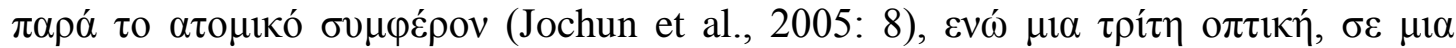

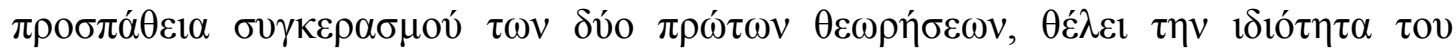

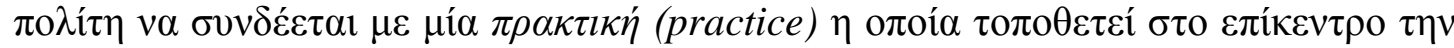

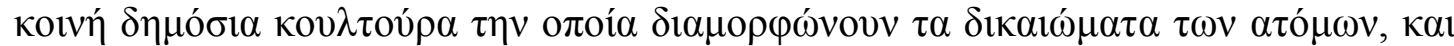

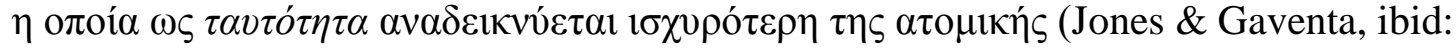

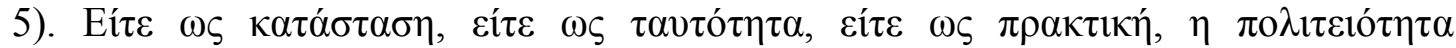

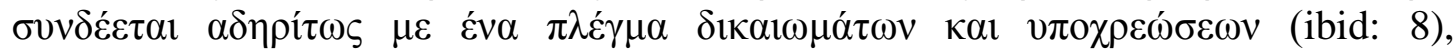

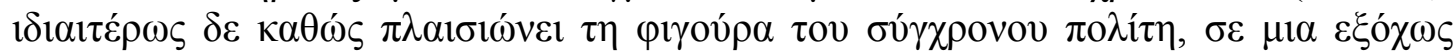

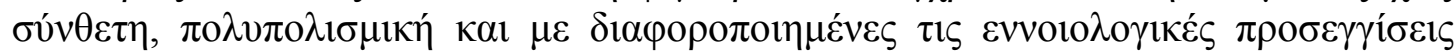

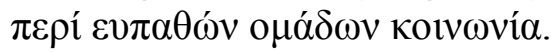

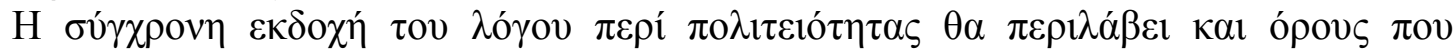

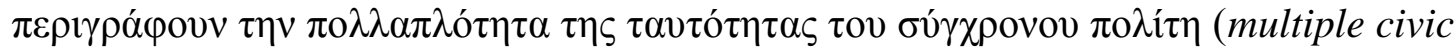

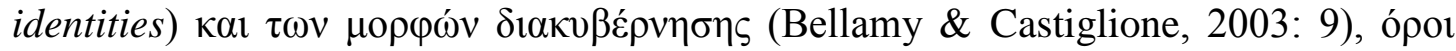

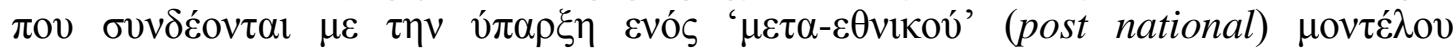

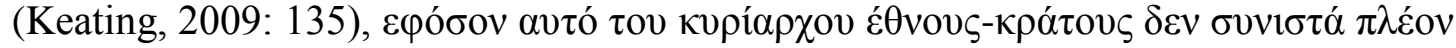

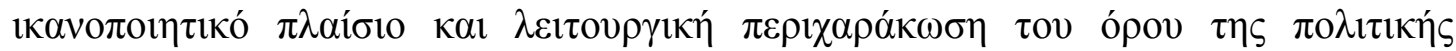

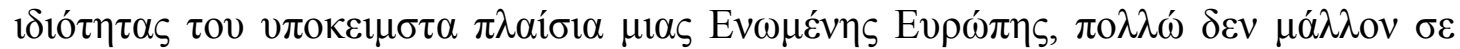

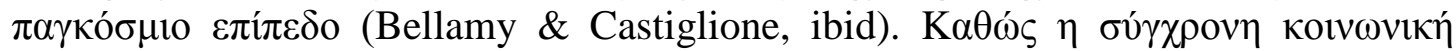

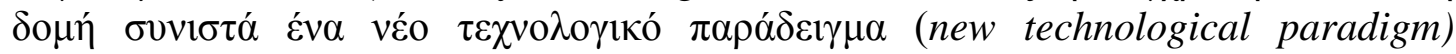

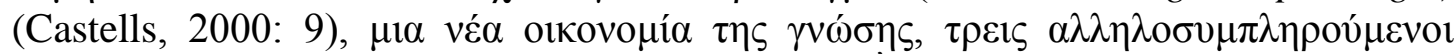

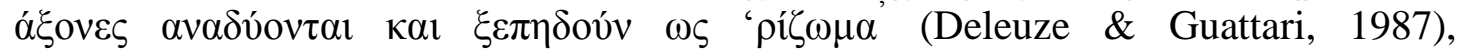

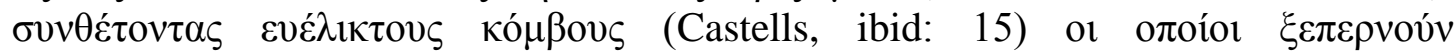

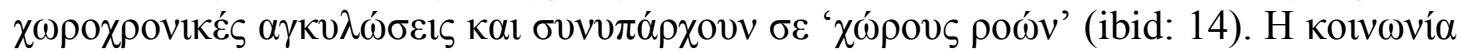

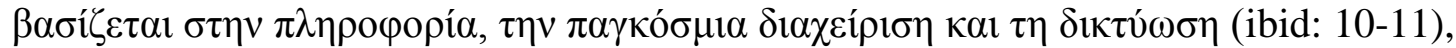

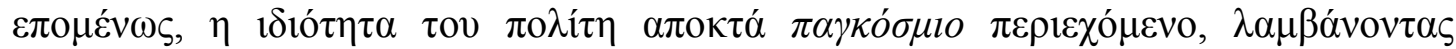

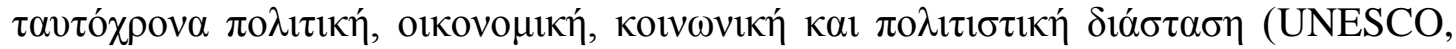

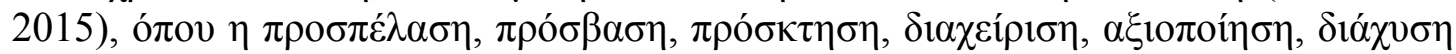

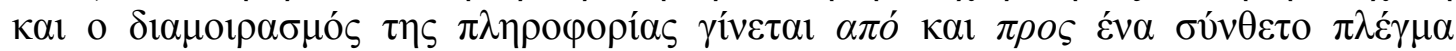

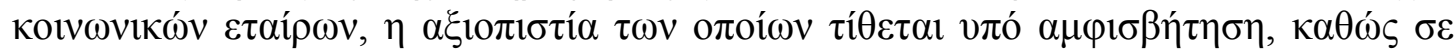

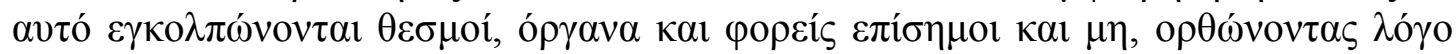

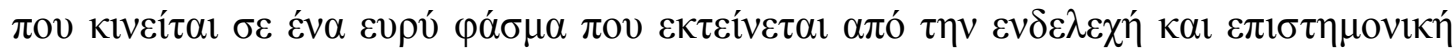

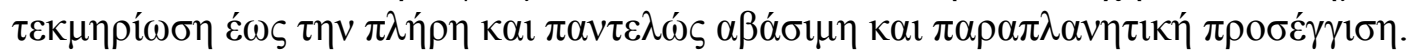

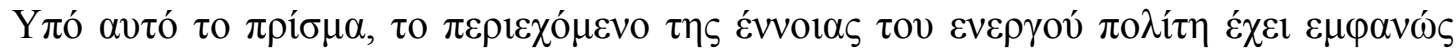

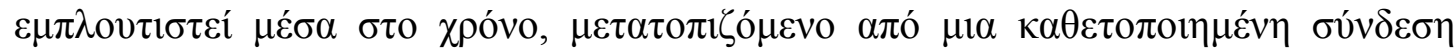

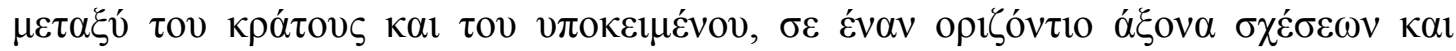

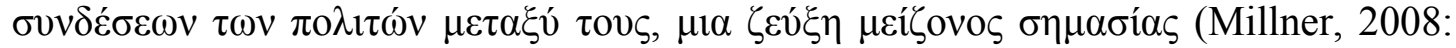

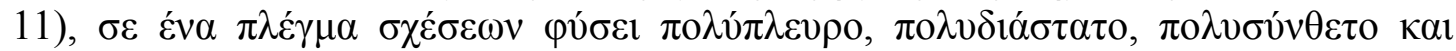

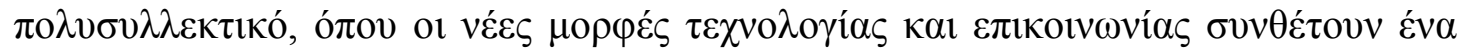

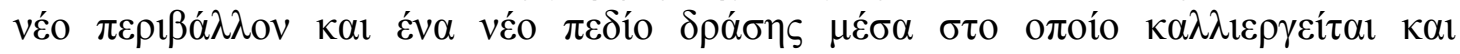

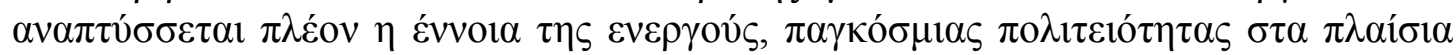

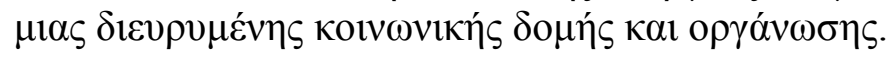

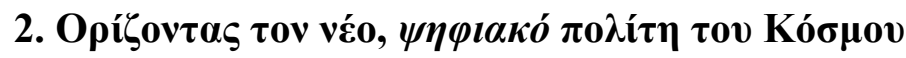

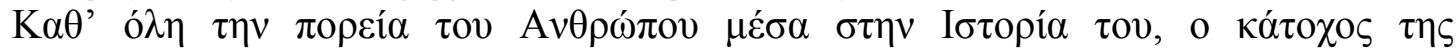

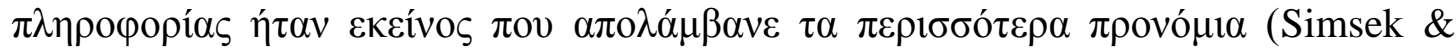




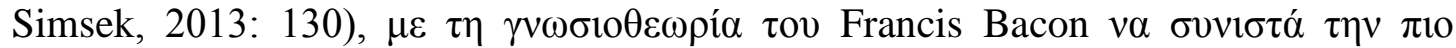

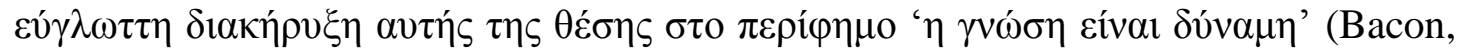

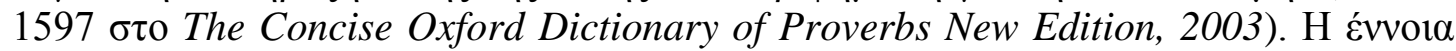

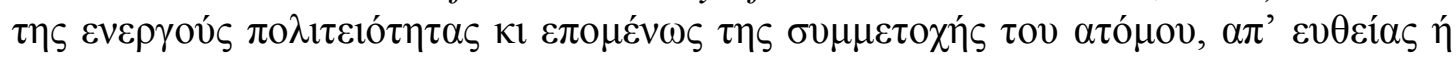

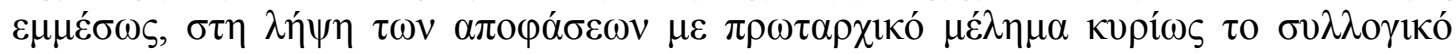

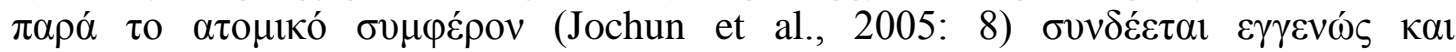

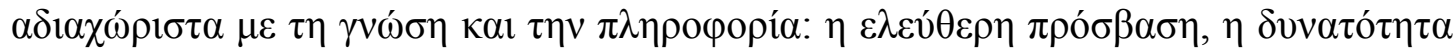

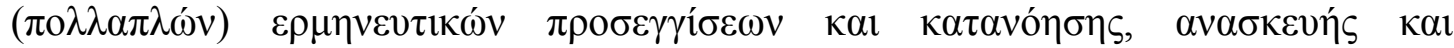

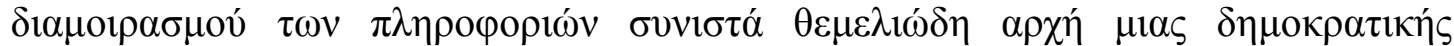

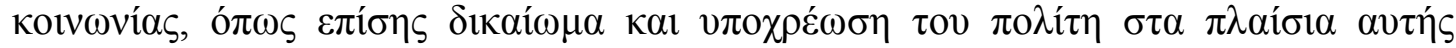
(Simsek \& Simsek, 2013: 126).

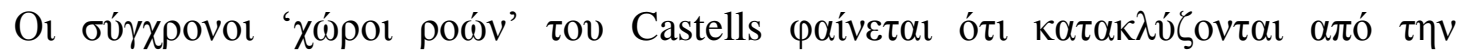

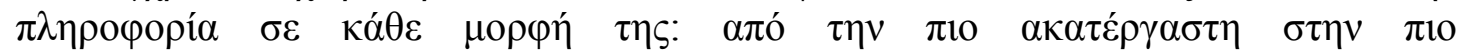

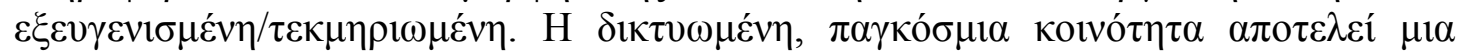

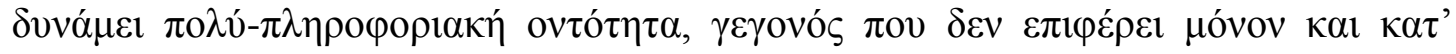

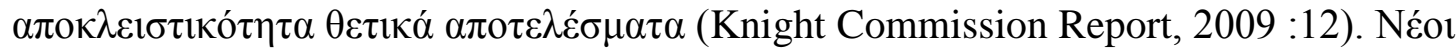

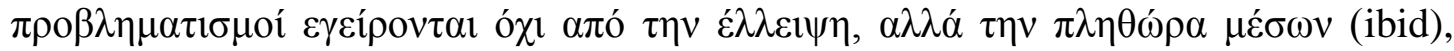

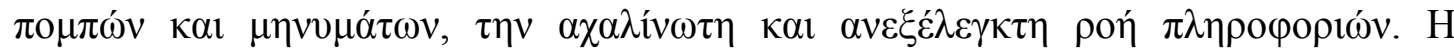

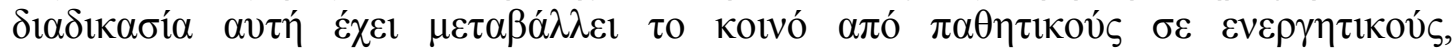

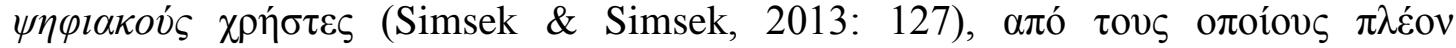

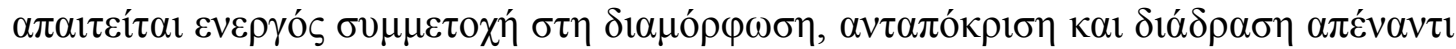

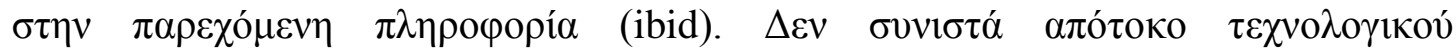

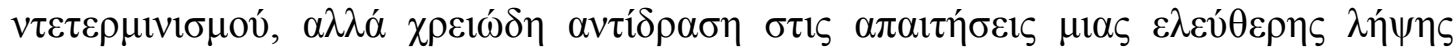

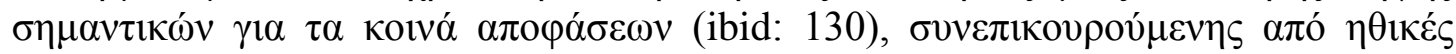

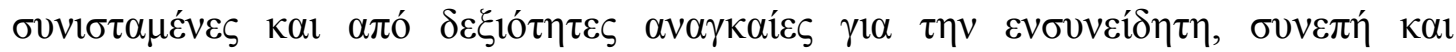

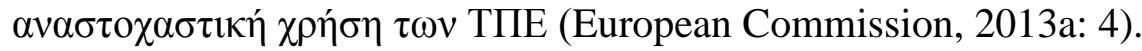

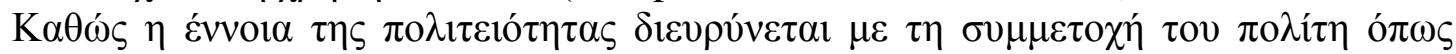

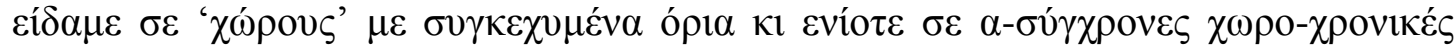

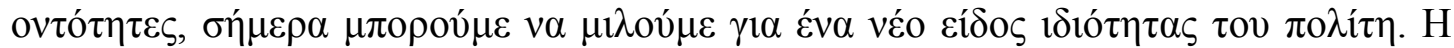

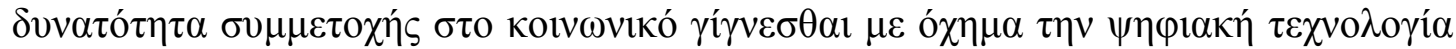

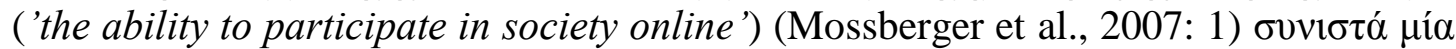

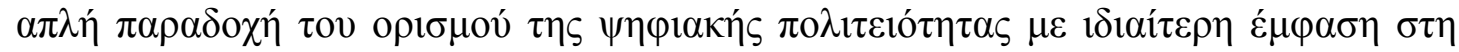

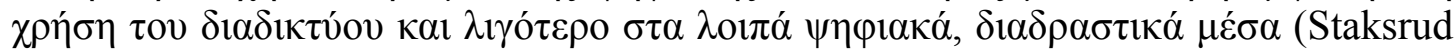

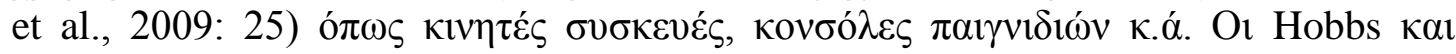

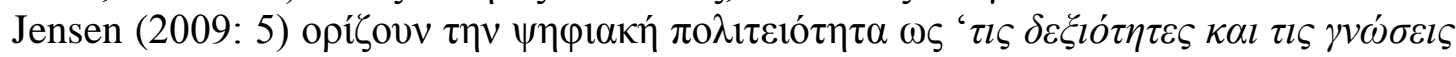

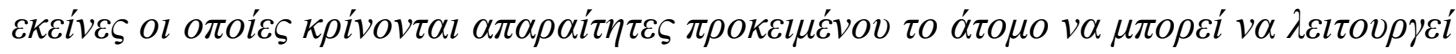

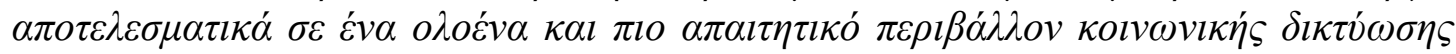

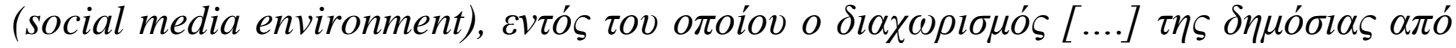

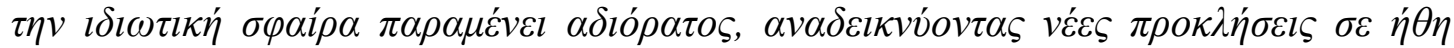

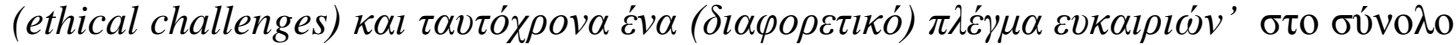

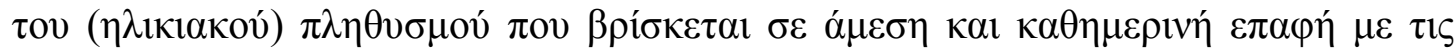

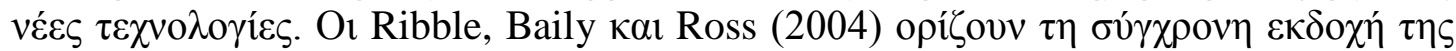

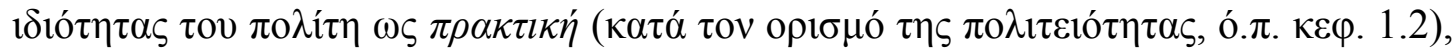

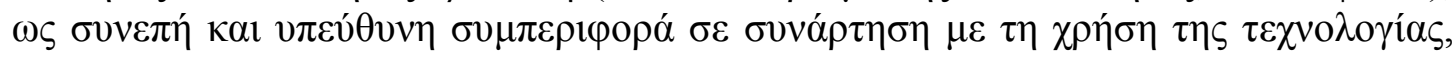

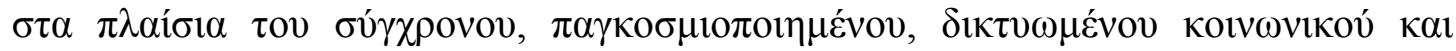

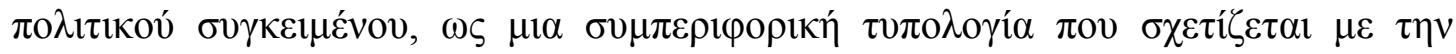

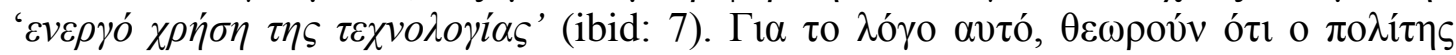

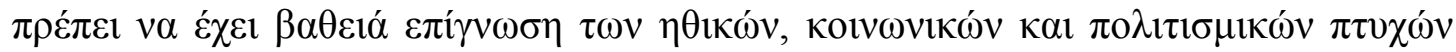

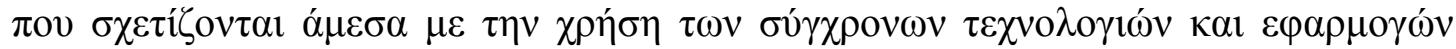
(ibid). 


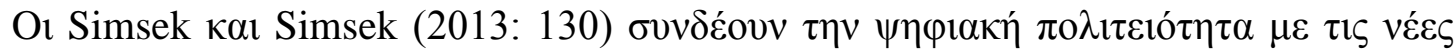
$\mu о \rho \varphi \varepsilon ́ \varsigma$ ( $\pi \lambda \alpha i ́ \sigma 10$, ó о

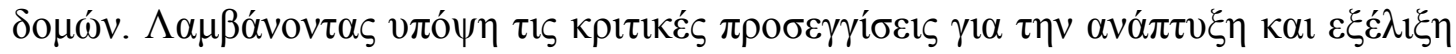

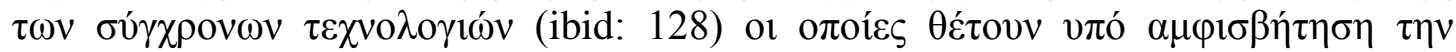

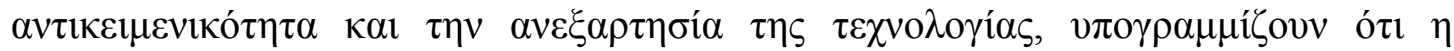

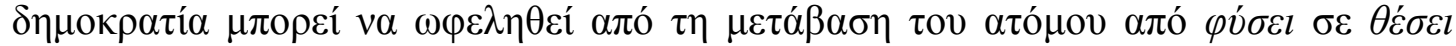

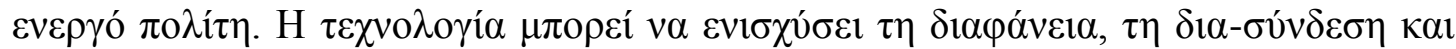

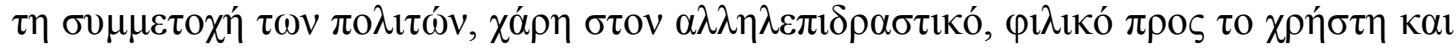

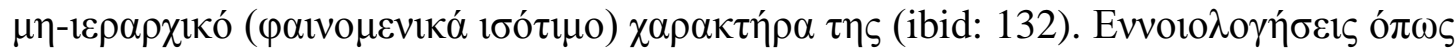

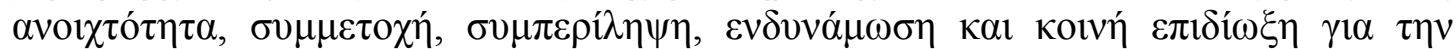

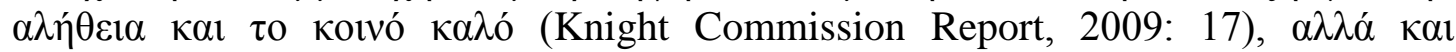

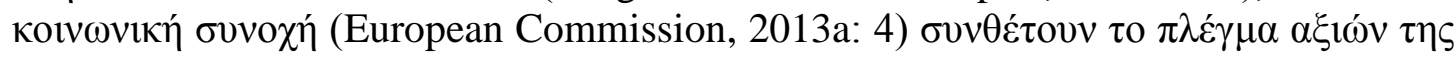

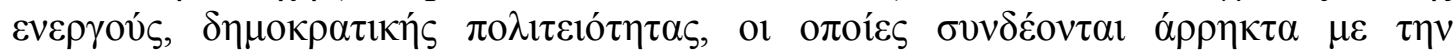

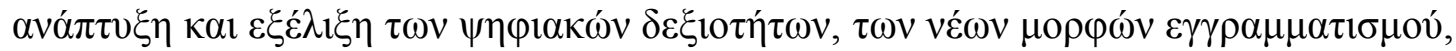

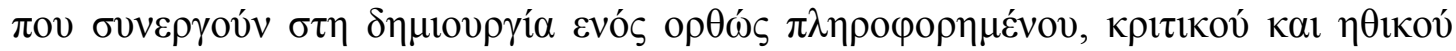
৩локєце́vov (Livingstone et al., 2008: 4).

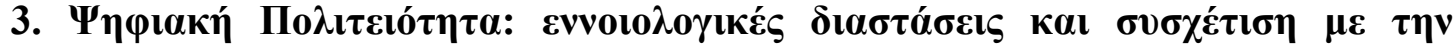

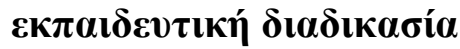

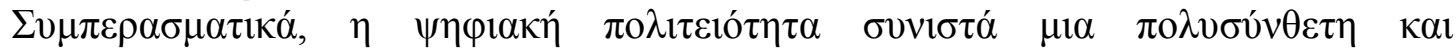

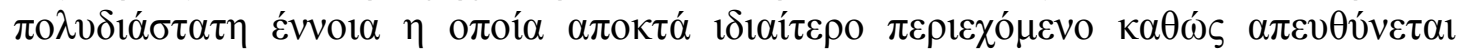

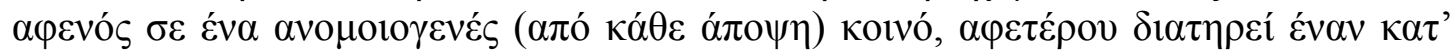

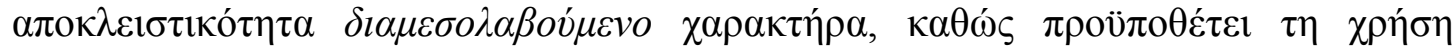

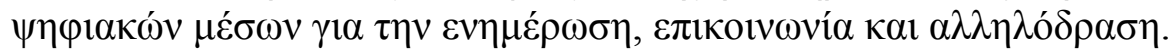

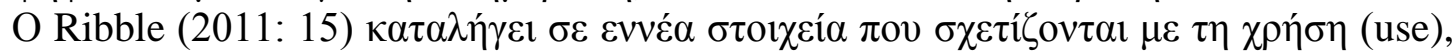

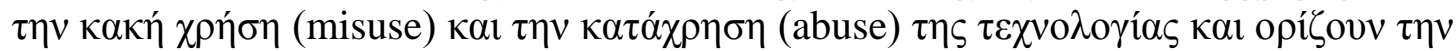

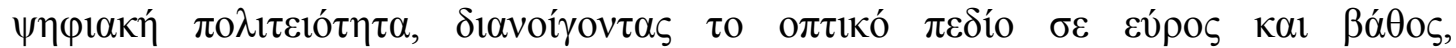

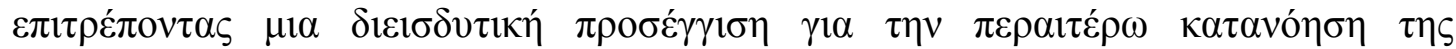

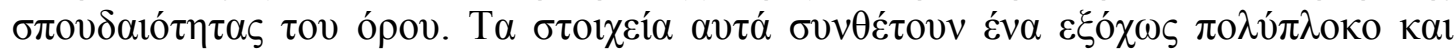

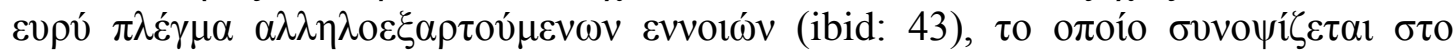

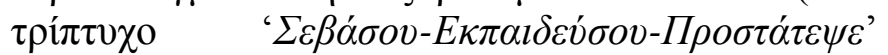

('Respect-Educate-Protect')

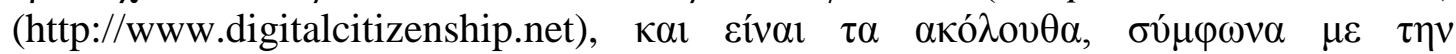

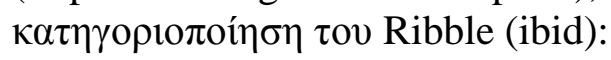

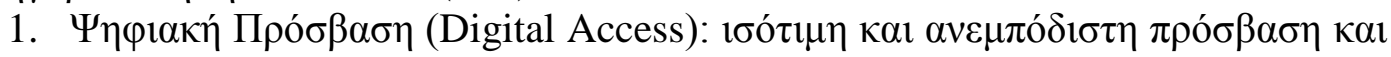

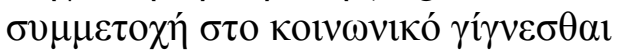

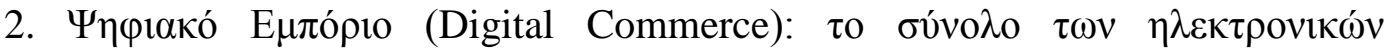

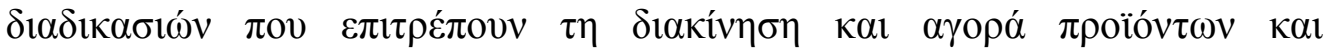

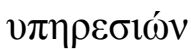

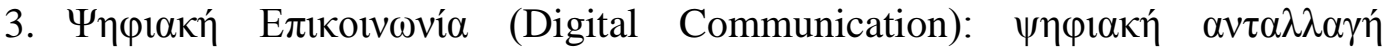

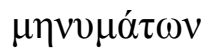

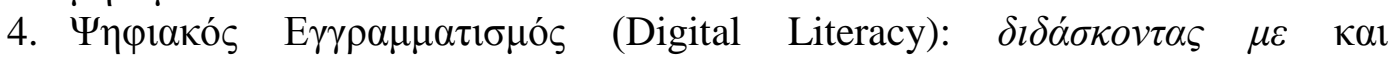

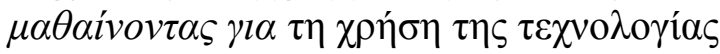

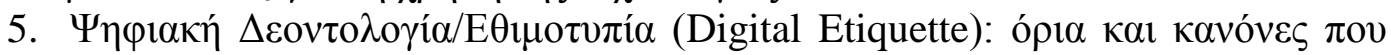

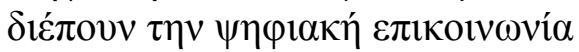

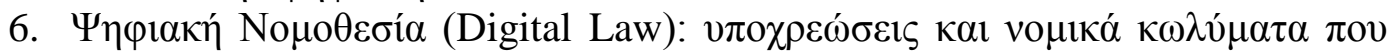

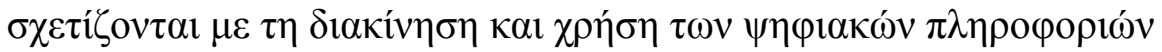

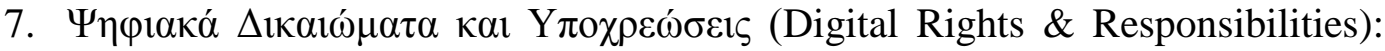

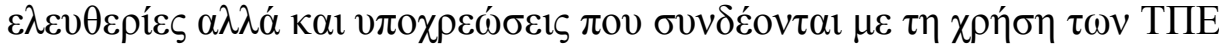




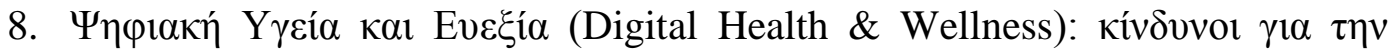

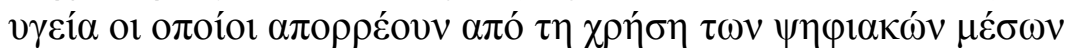

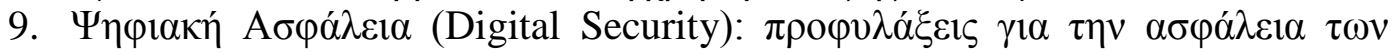

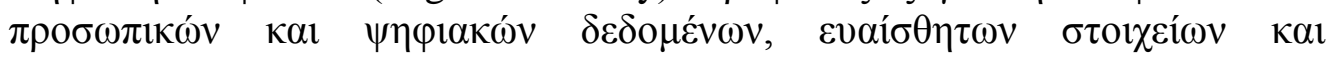

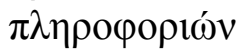

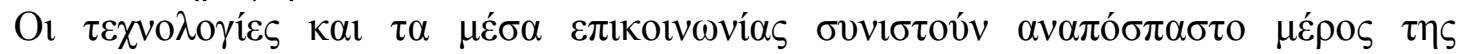

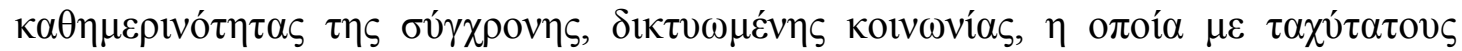

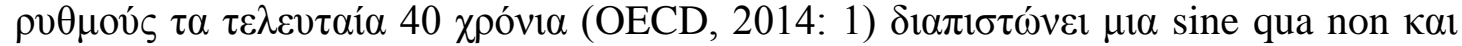

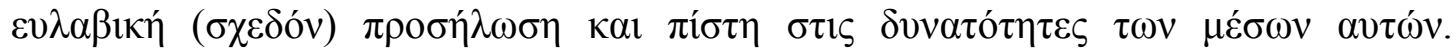

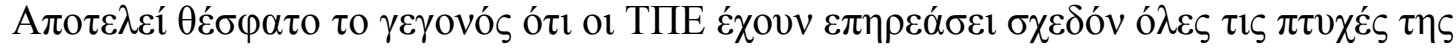

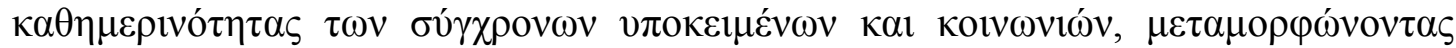

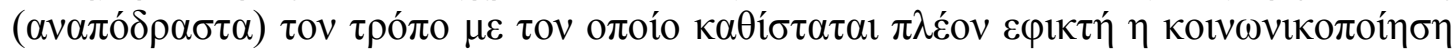

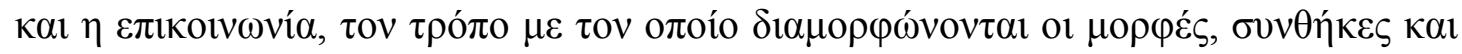
$\sigma \chi \varepsilon \dot{\sigma \varepsilon \varepsilon 1 \varsigma} \varepsilon \rho \gamma \alpha \sigma i \alpha \varsigma$ (ibid).

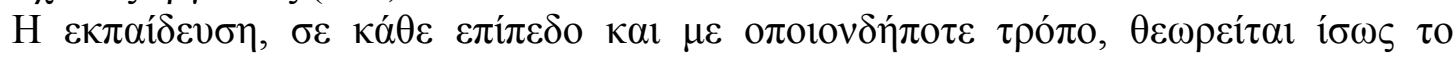

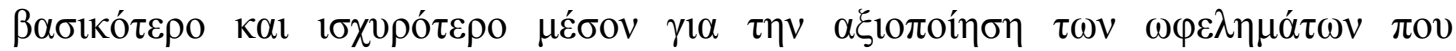

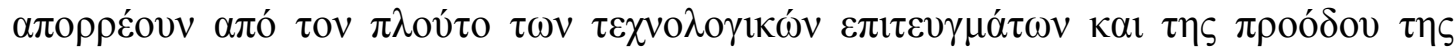

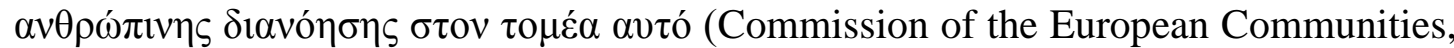

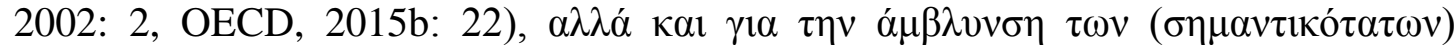

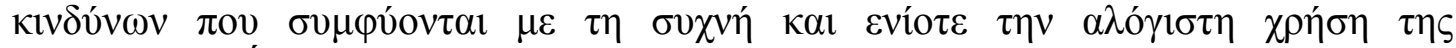

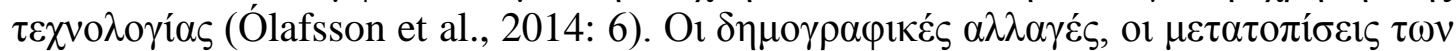

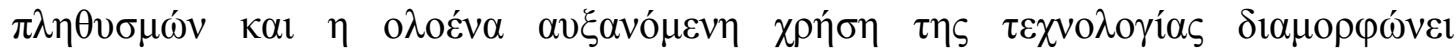

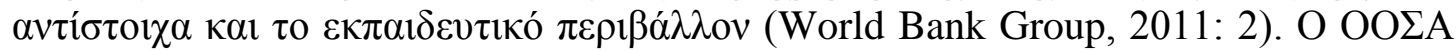

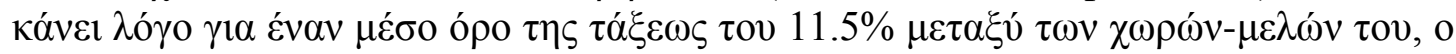

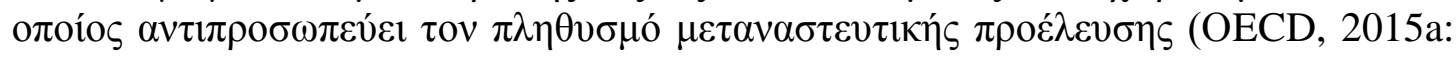

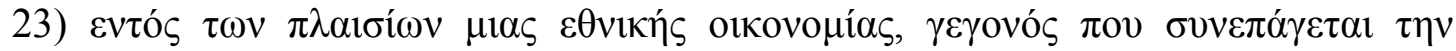

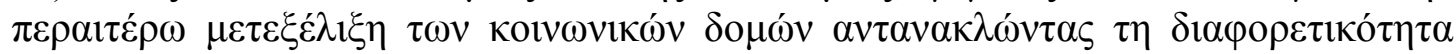

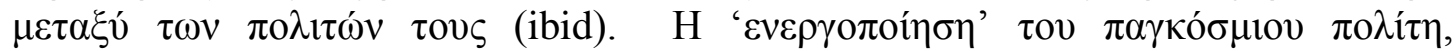

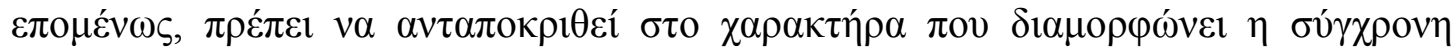

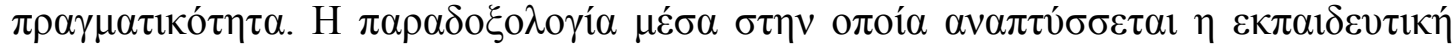

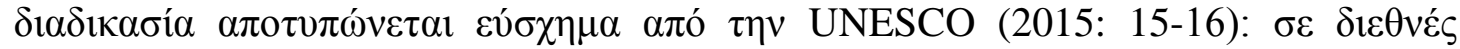

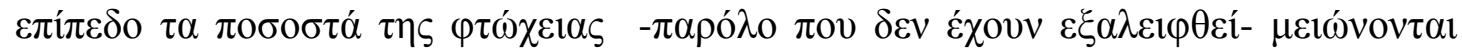

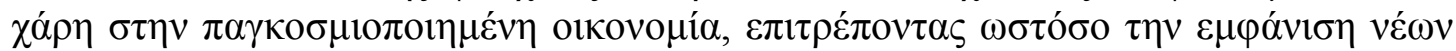

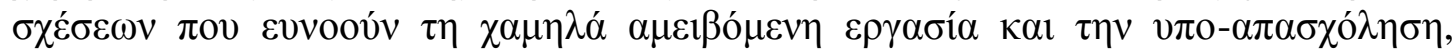

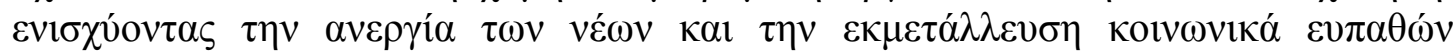

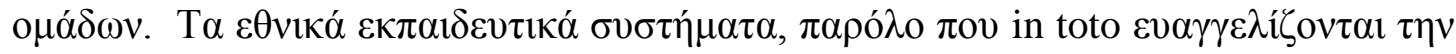

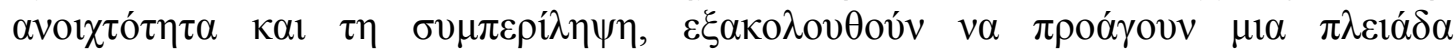

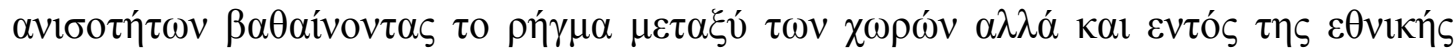

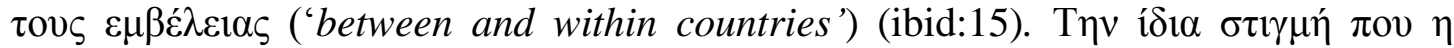

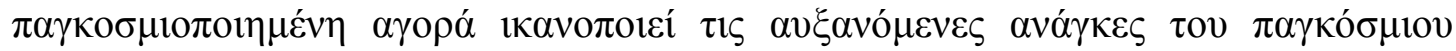

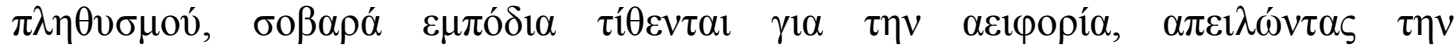

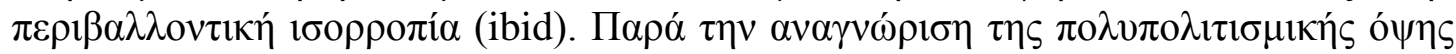

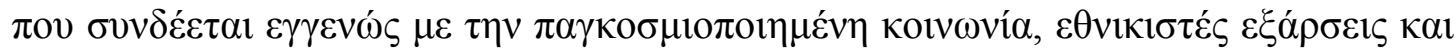

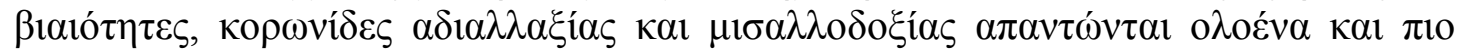

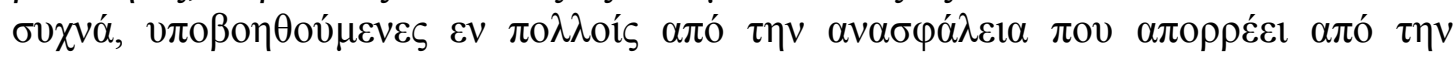

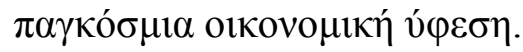

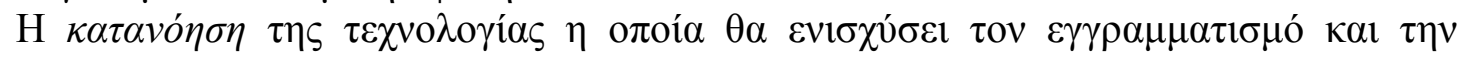

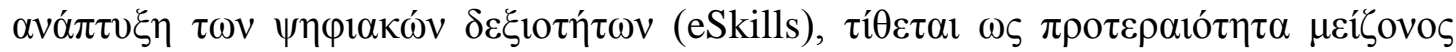

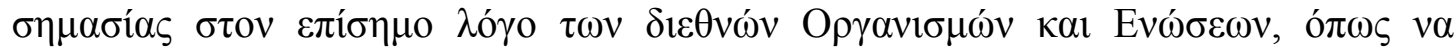

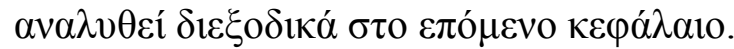




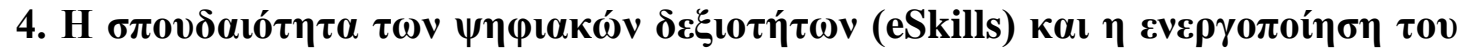

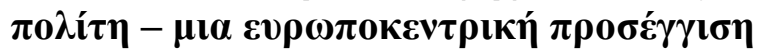

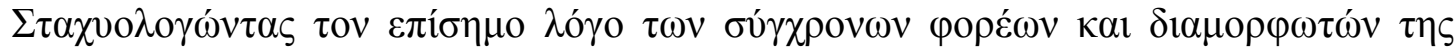

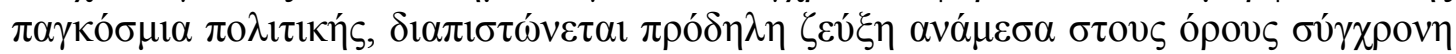

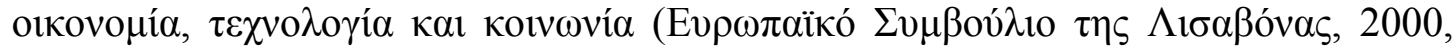

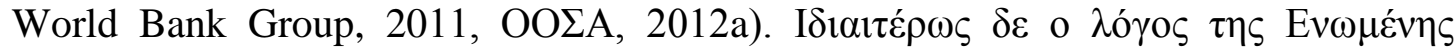

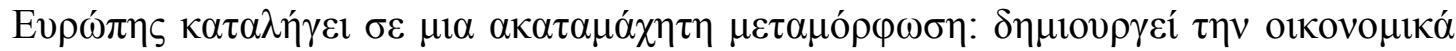

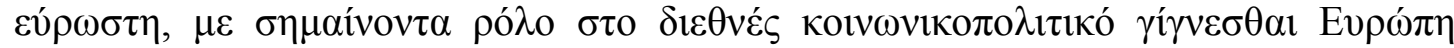

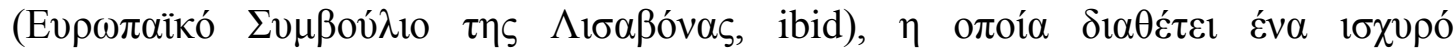

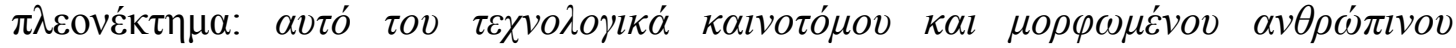

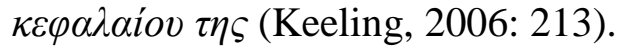

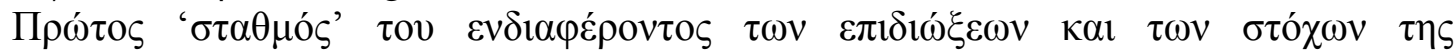

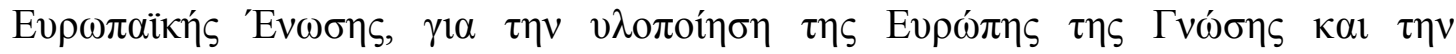

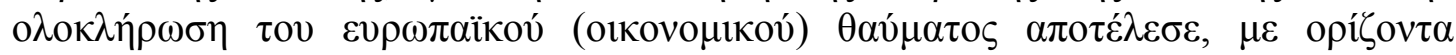

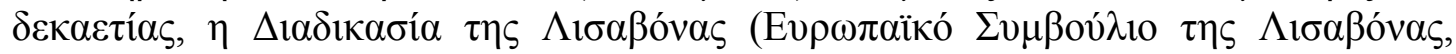

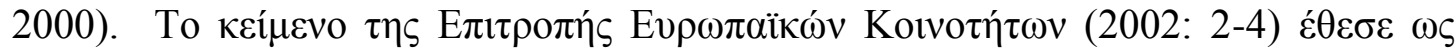

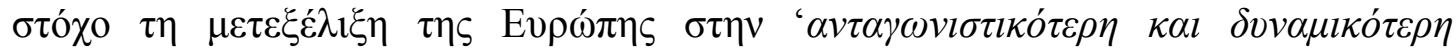

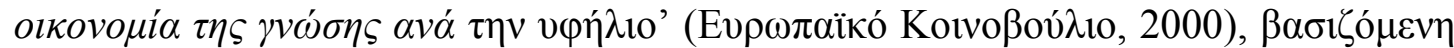

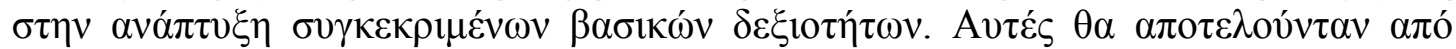

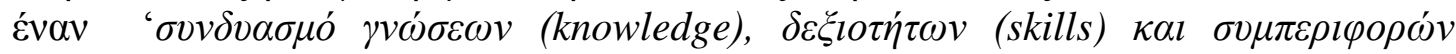

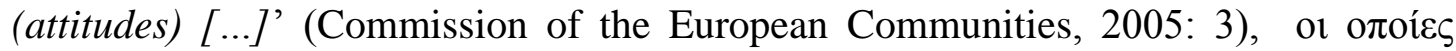

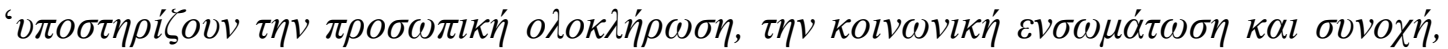

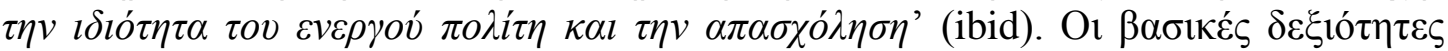

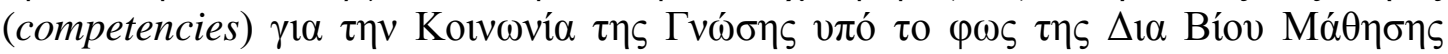

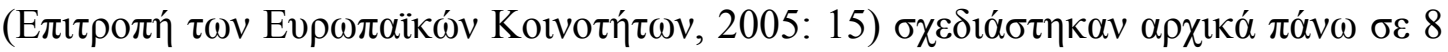

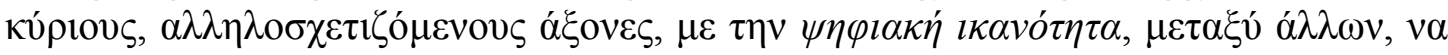

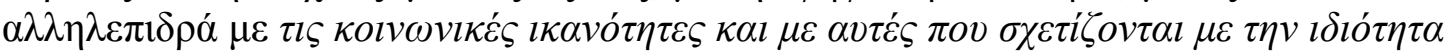

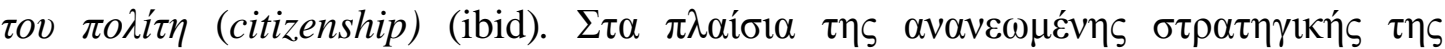

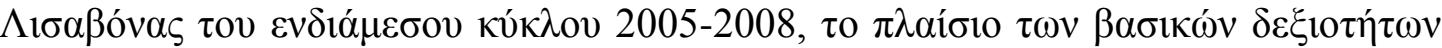

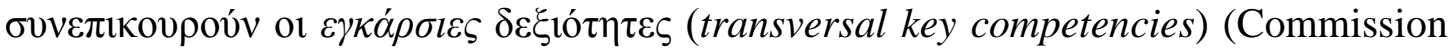

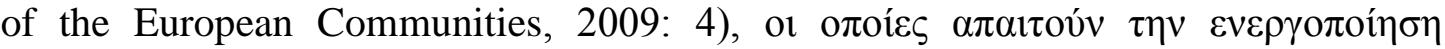

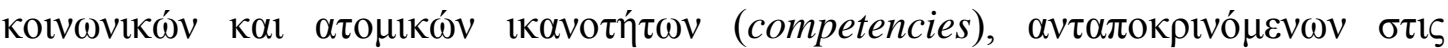

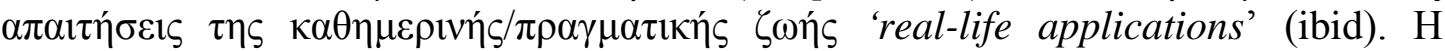

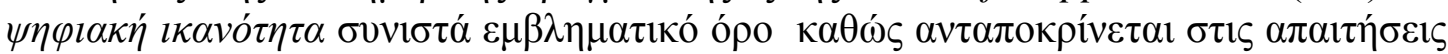

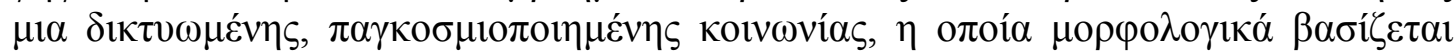

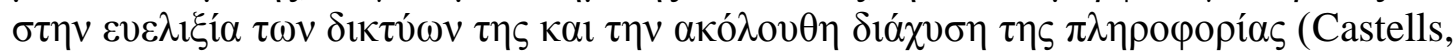

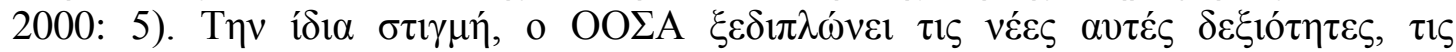

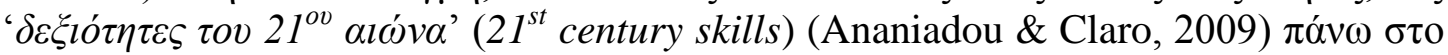

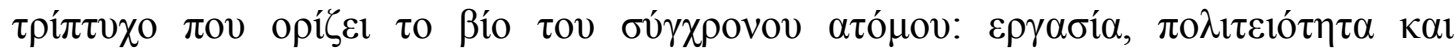

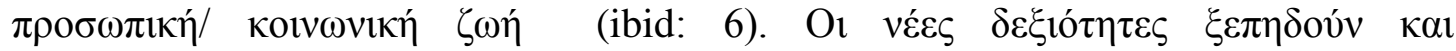

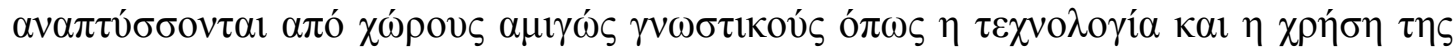

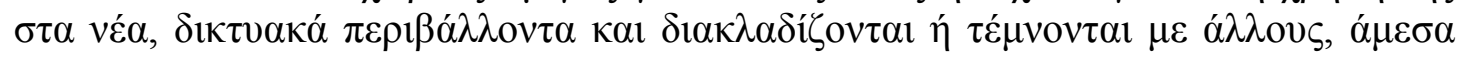

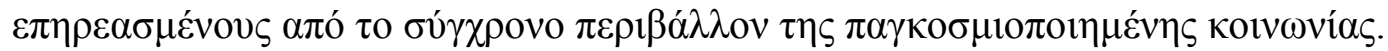

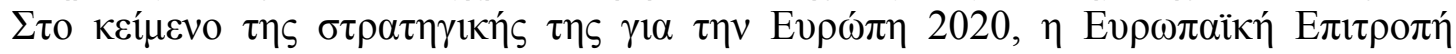

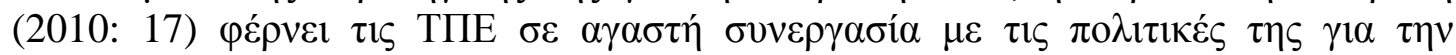

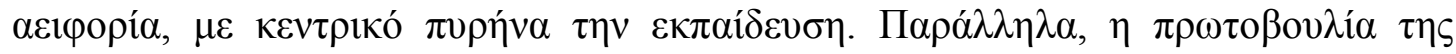

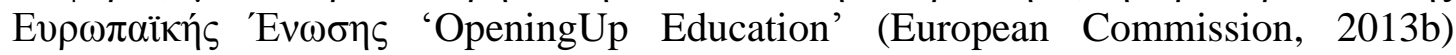

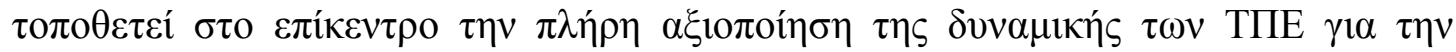

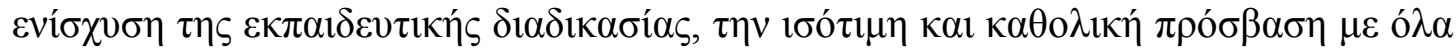

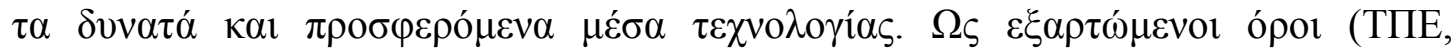




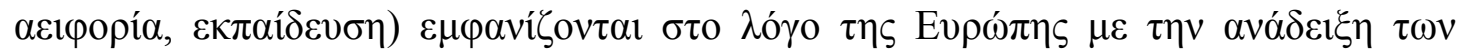

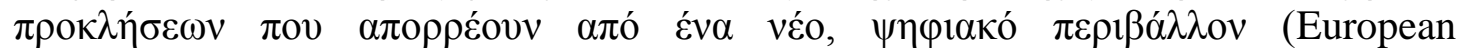

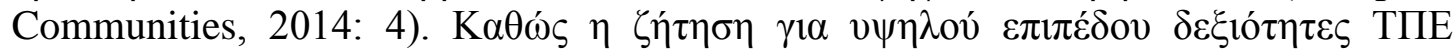

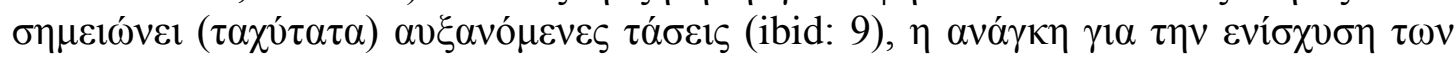

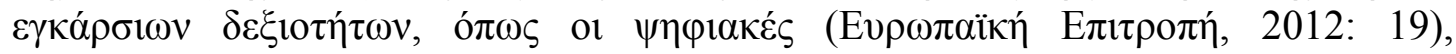

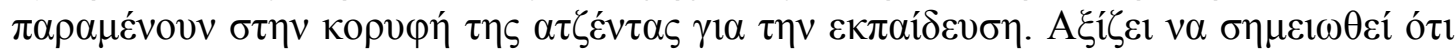

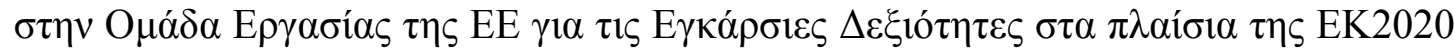

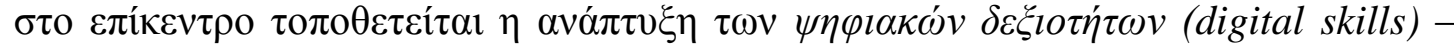

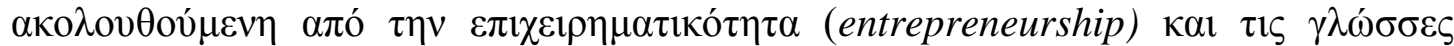
(languages) (European Commission/ET2020 Working Group on Transversal Skills, 2014).

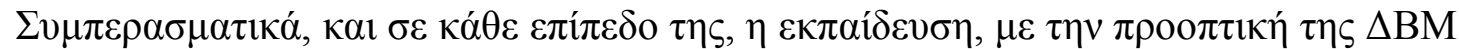

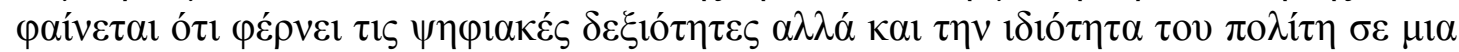

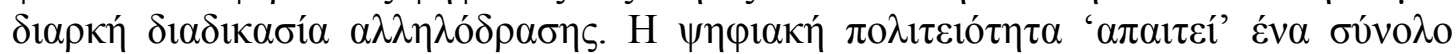

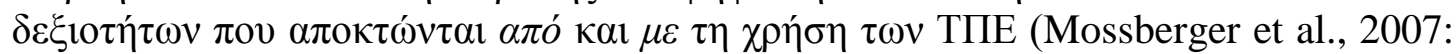

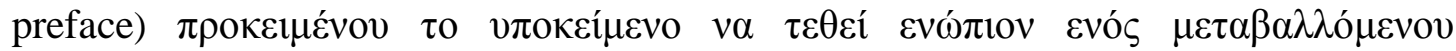

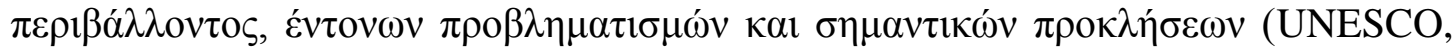

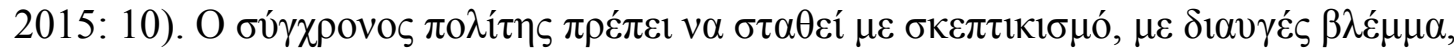

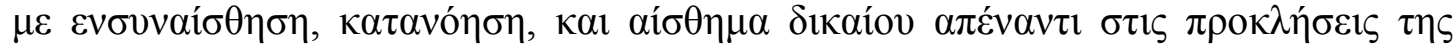

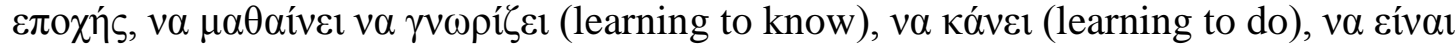
(learning to be) $\kappa \alpha \imath v \alpha \sigma \nu \mu \beta \iota \omega ́ v \varepsilon \imath$ (learning to live together) (UNESCO, ibid: 39).

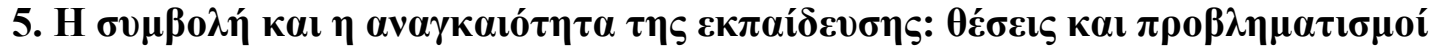

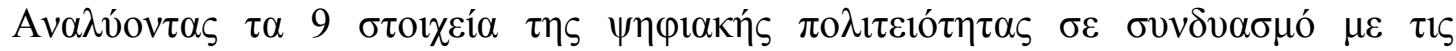

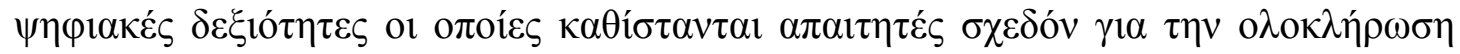

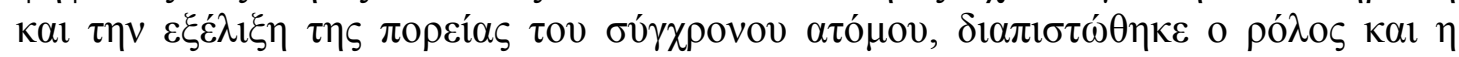

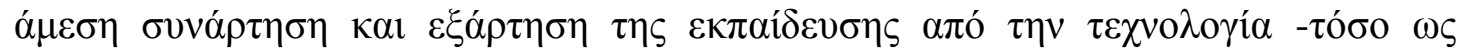

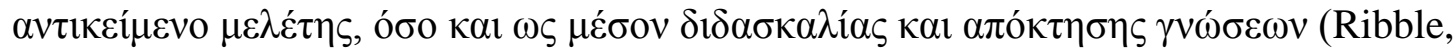

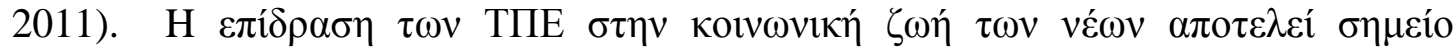

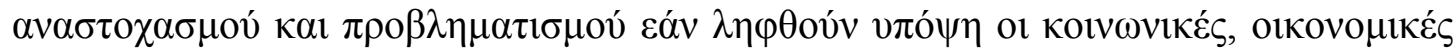

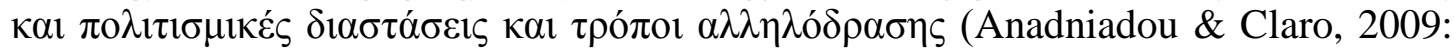

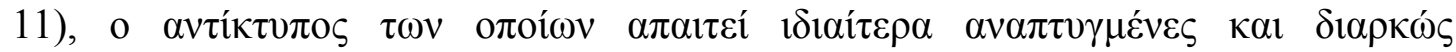
$\varepsilon \xi \varepsilon \lambda ı \sigma \sigma o ́ \mu \varepsilon v \varepsilon \varsigma \delta \varepsilon \xi$ ló $\eta \tau \varepsilon \varsigma$.

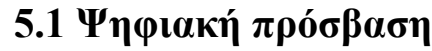

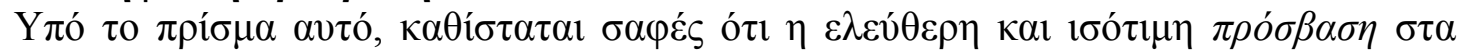

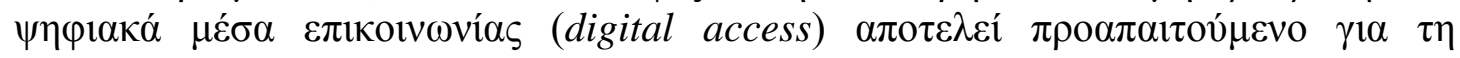

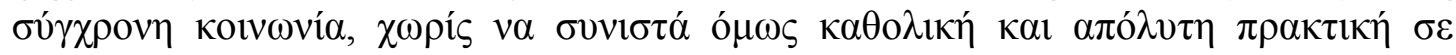

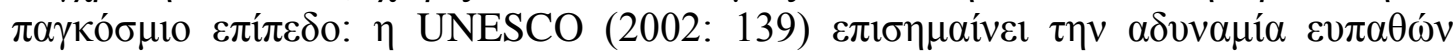

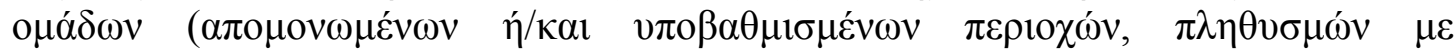

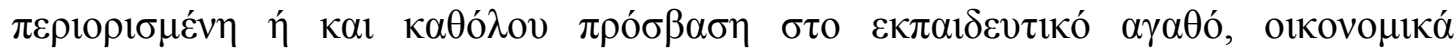

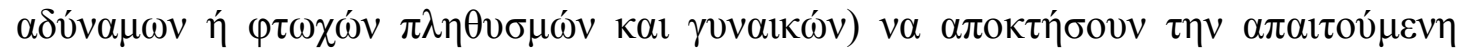

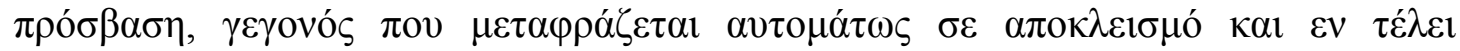

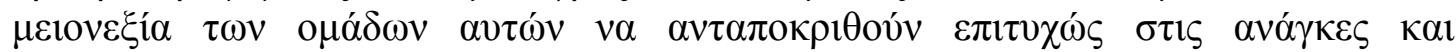

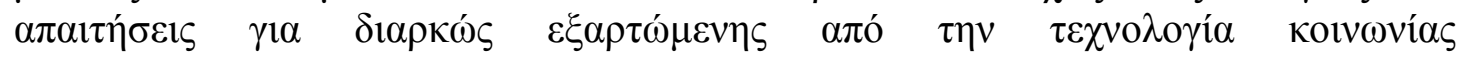

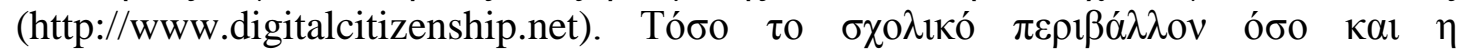

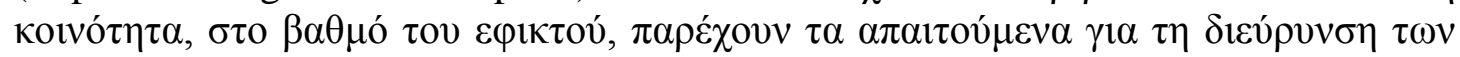

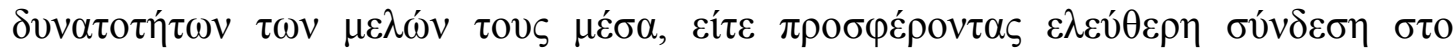

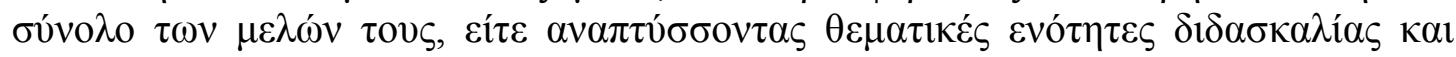

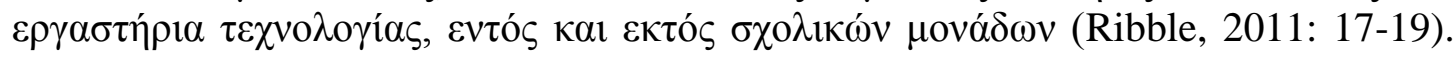




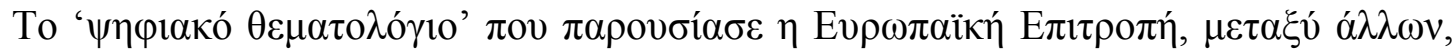

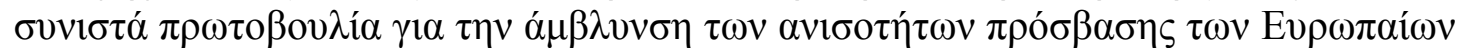

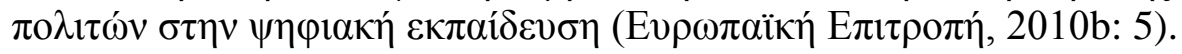

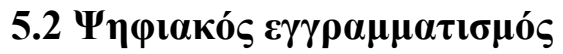

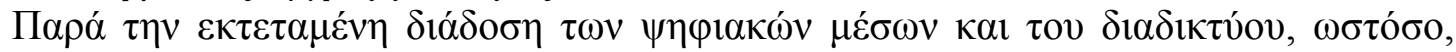

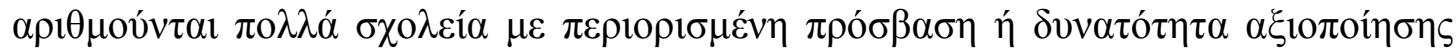

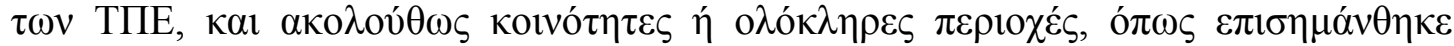

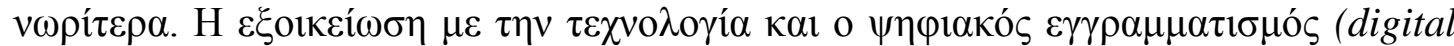

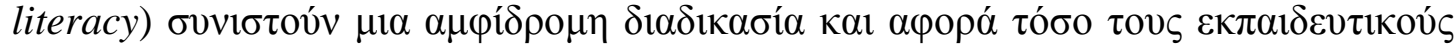

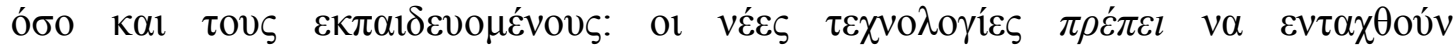

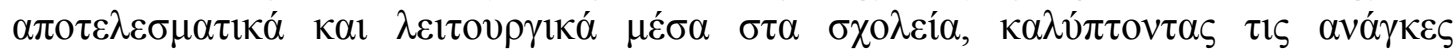

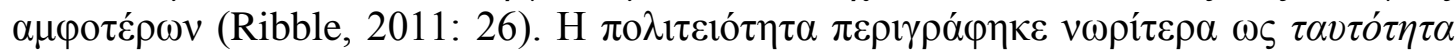

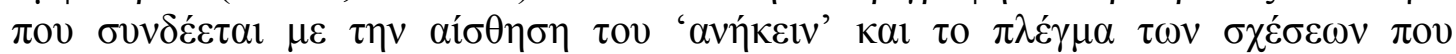

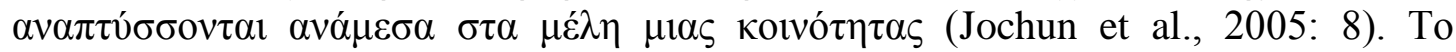

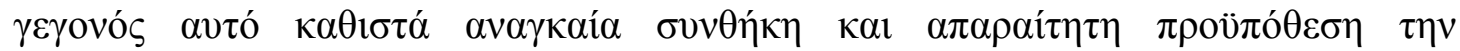

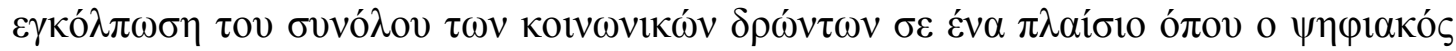

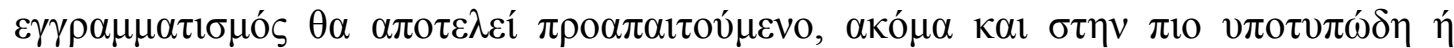

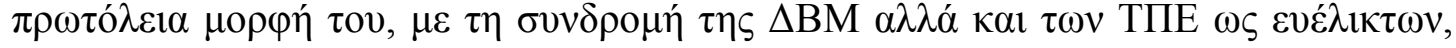
$\varepsilon \kappa \pi \alpha 1 \delta \varepsilon v \tau 1 \kappa \omega ́ v ~ \mu \varepsilon ́ \sigma \omega v$.

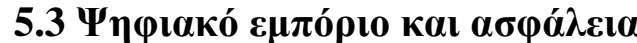

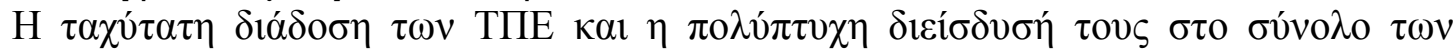

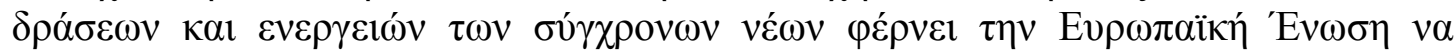

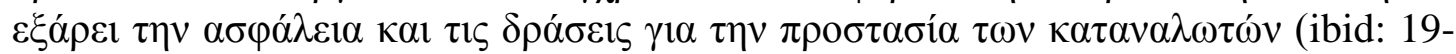

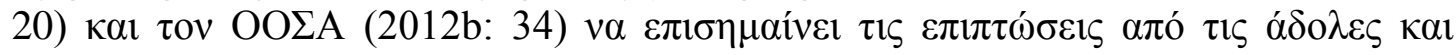

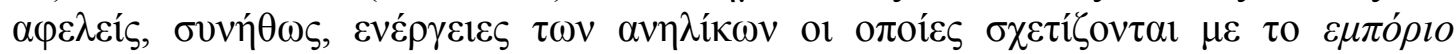

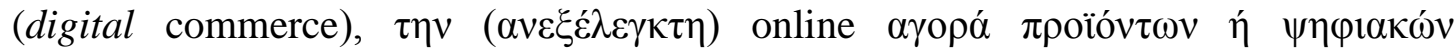

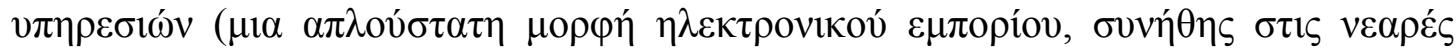
$\eta \lambda \iota \kappa i ́ \varepsilon s$ a

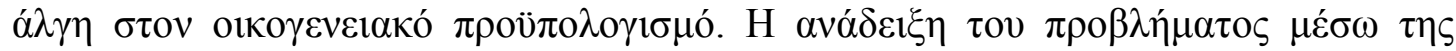

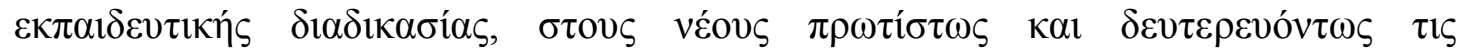

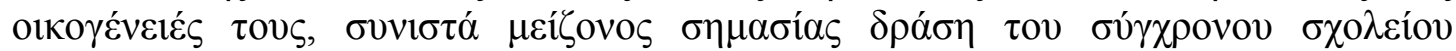

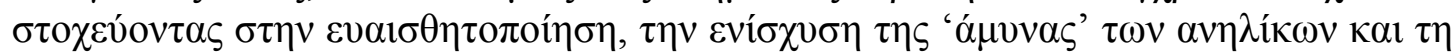

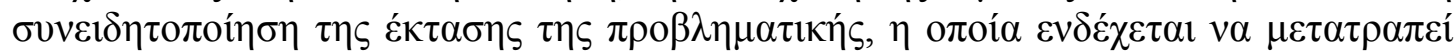

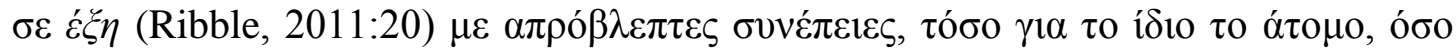

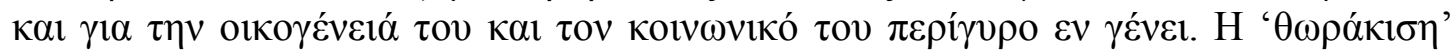

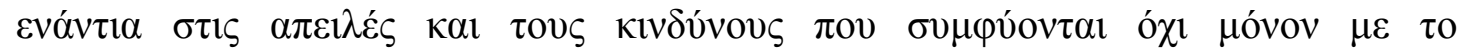

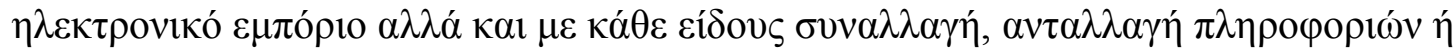

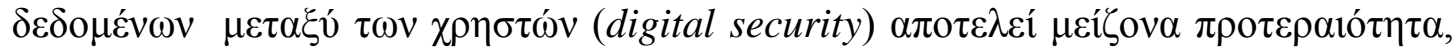

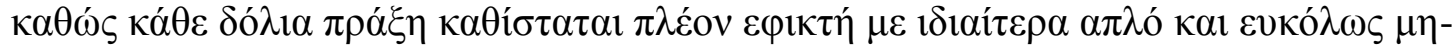
$\alpha v \imath \chi v \varepsilon v ́ \sigma u$ т

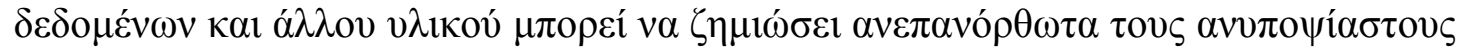

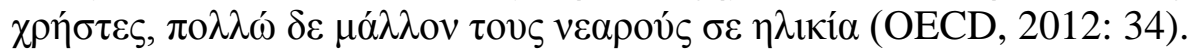

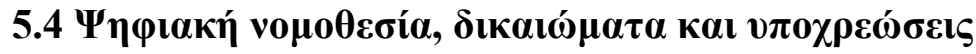

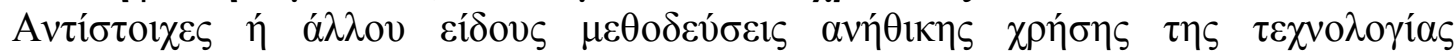

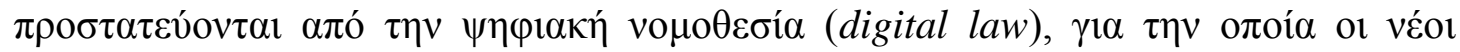

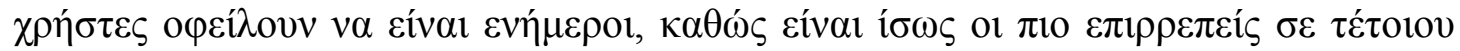

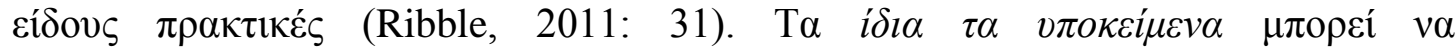




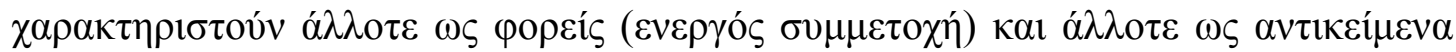

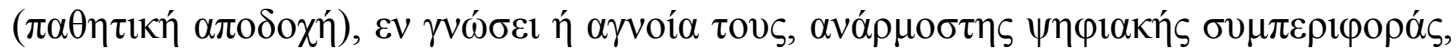

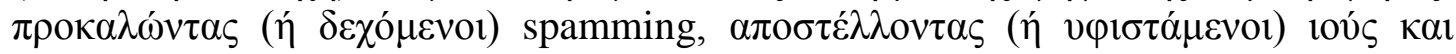

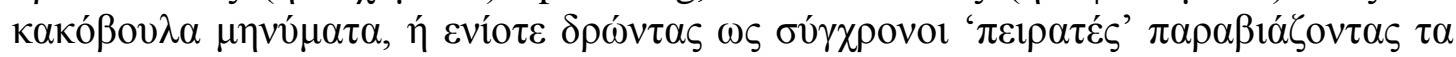

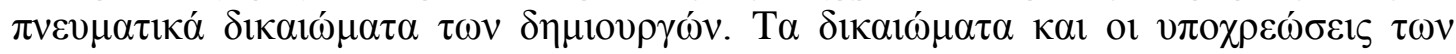

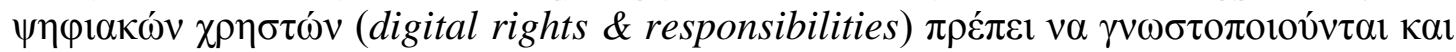

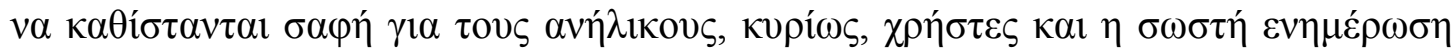

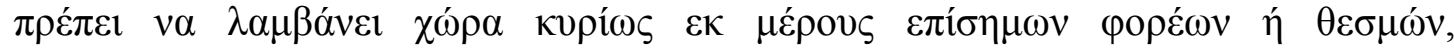

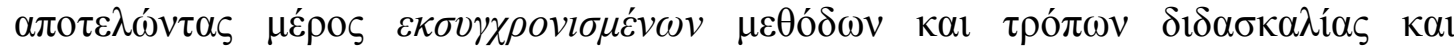

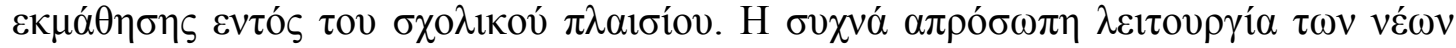

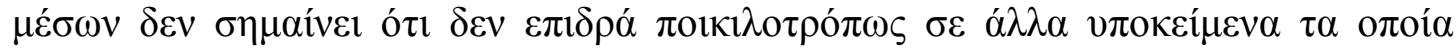

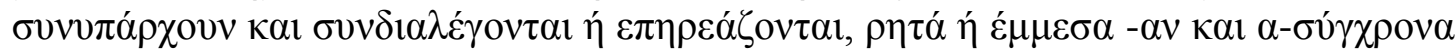

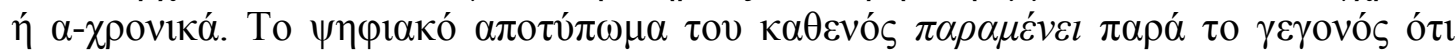

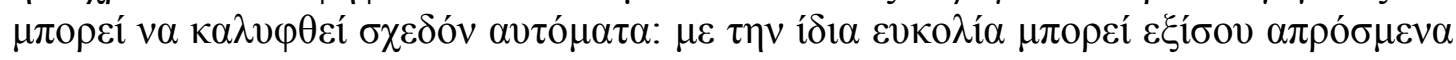

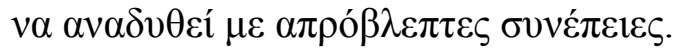

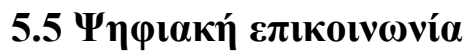

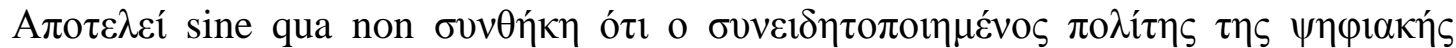

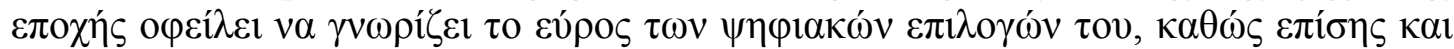

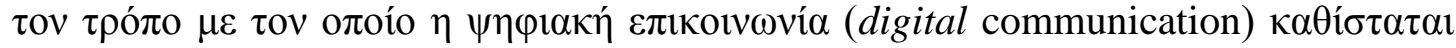

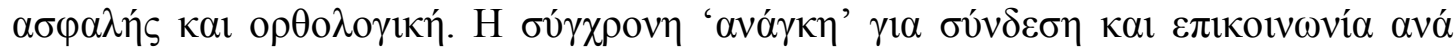

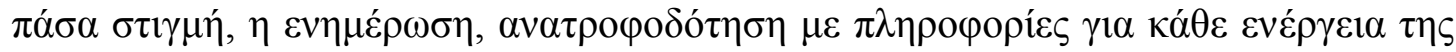

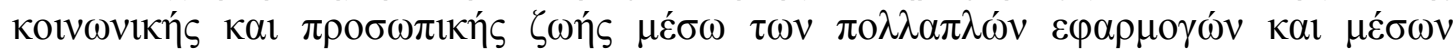

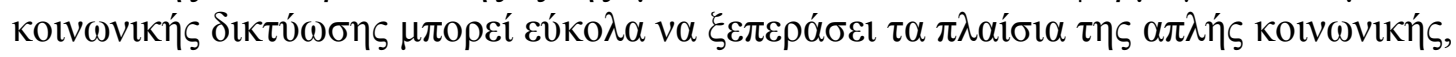

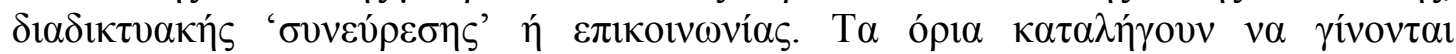

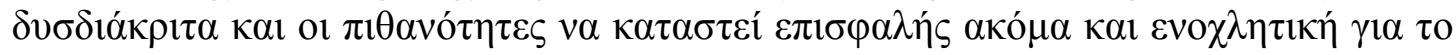

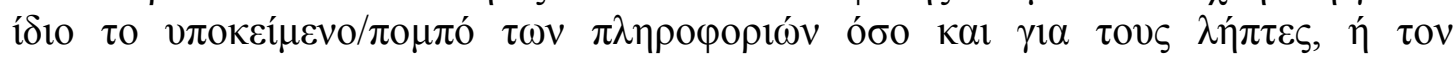

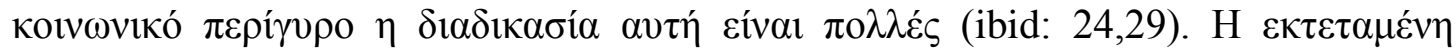

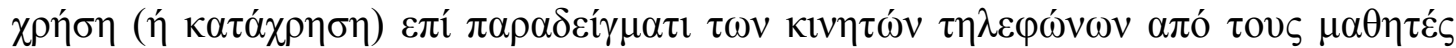

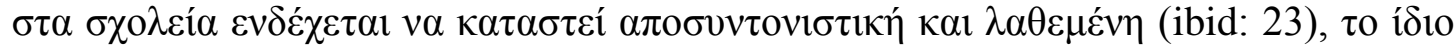

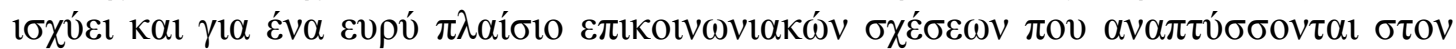

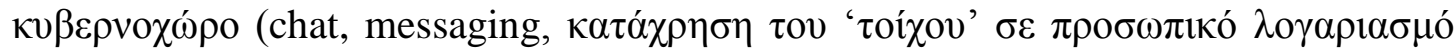

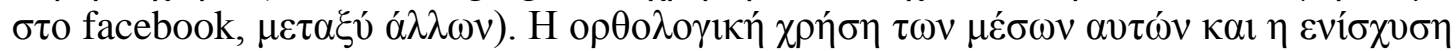

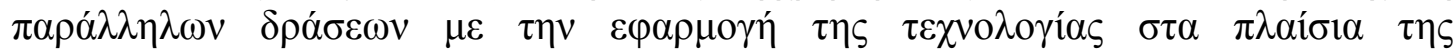

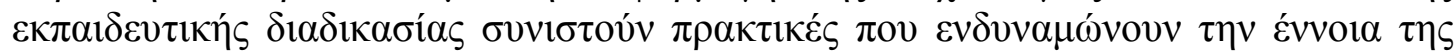

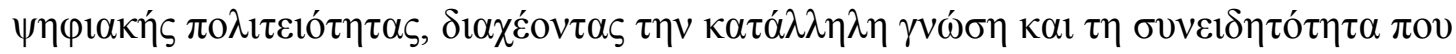

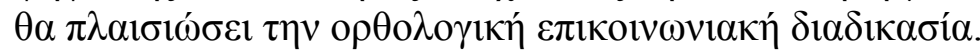

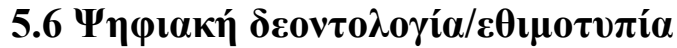

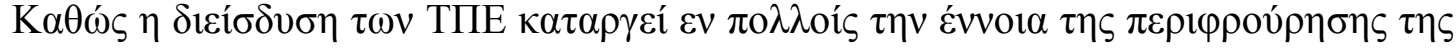

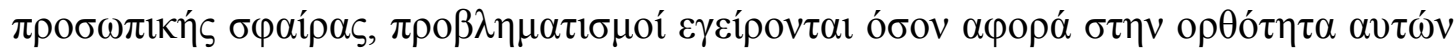

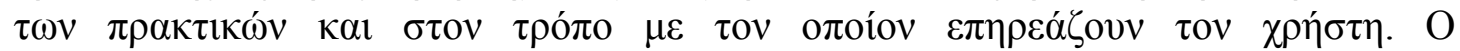

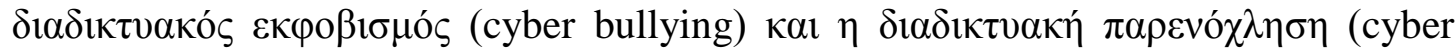

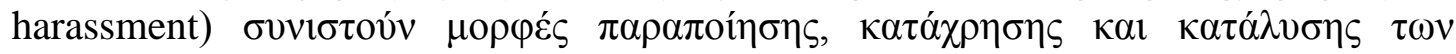

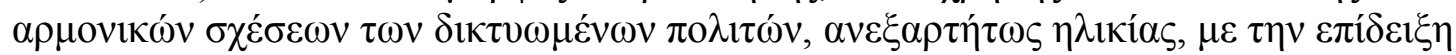

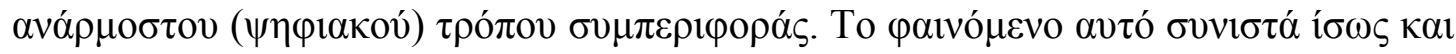

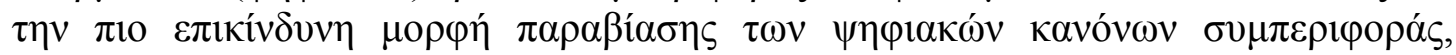

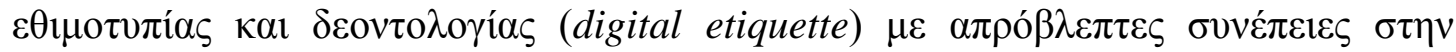

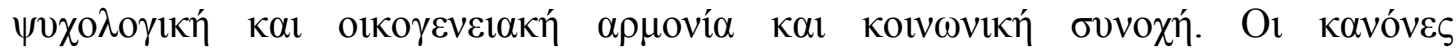

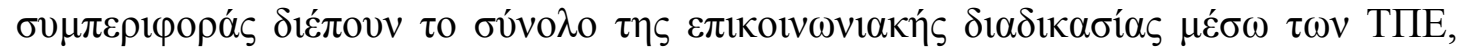




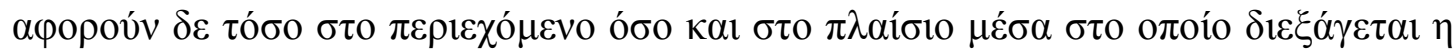

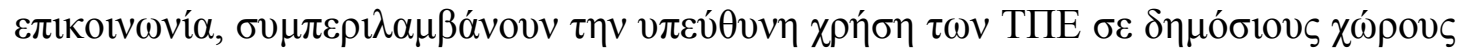

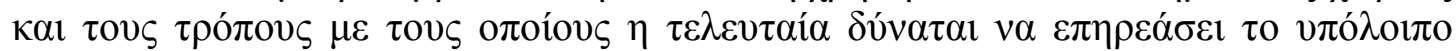

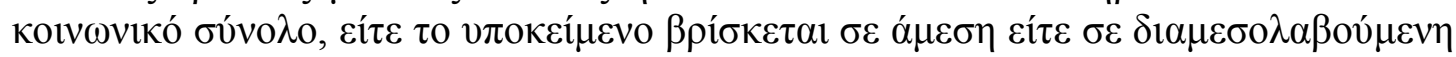

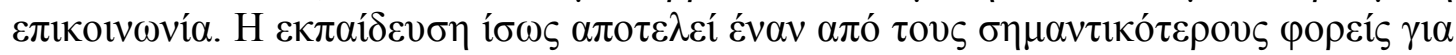

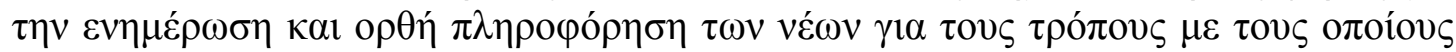

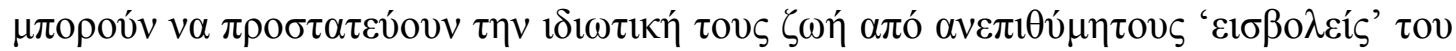

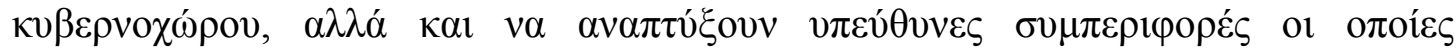

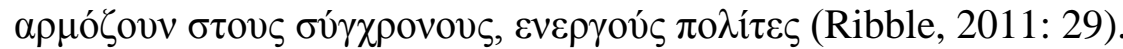

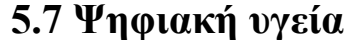

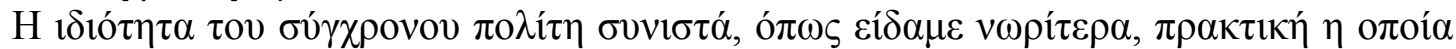

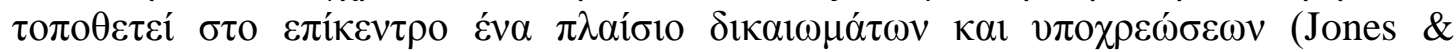

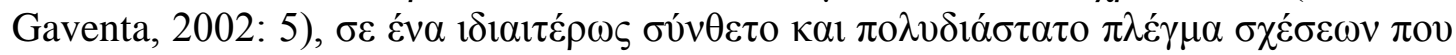
$\alpha v \alpha \pi \tau v ́ \sigma \sigma o v \tau \alpha$ l

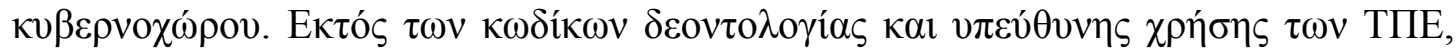

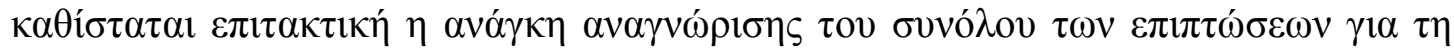

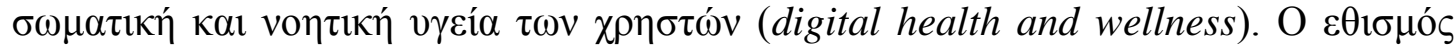

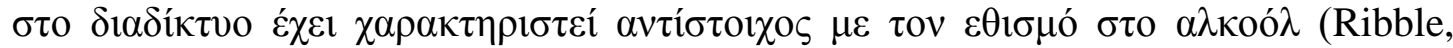

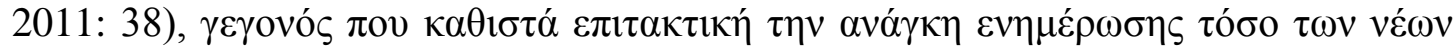

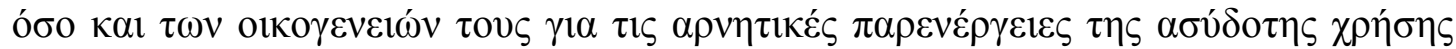

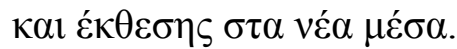

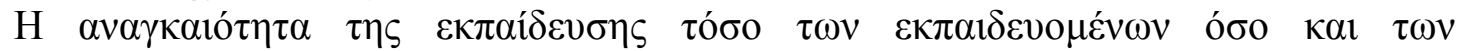

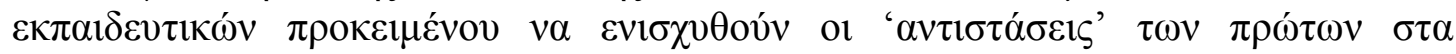

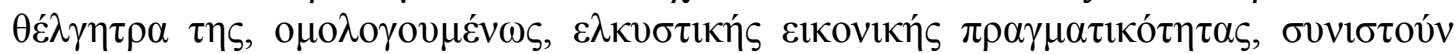

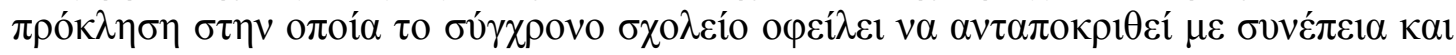

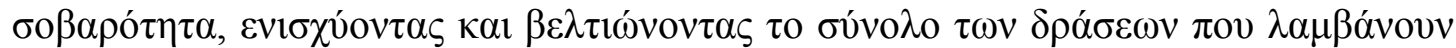

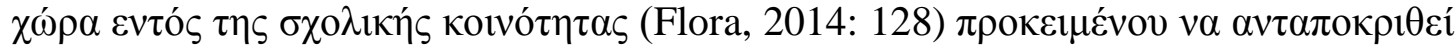

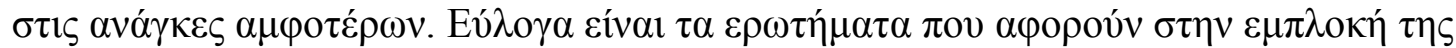

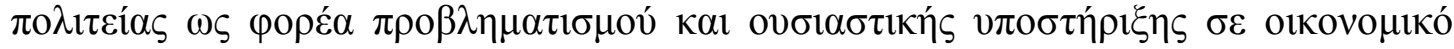

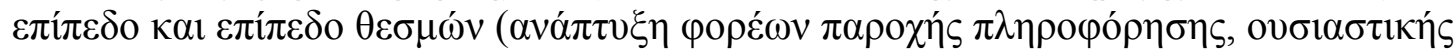
$\mu \varepsilon \dot{\rho} \mu \nu \alpha \varsigma \kappa \alpha$ vл

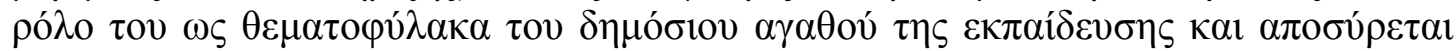

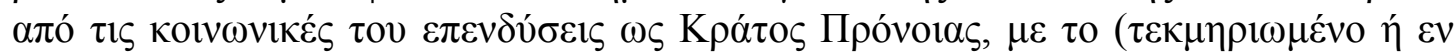

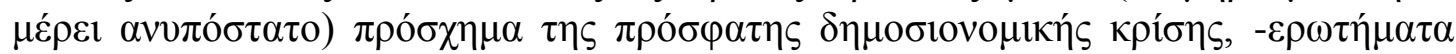

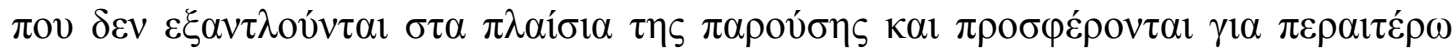
$\delta 1 \varepsilon \rho \varepsilon v ́ v \eta \sigma \eta \kappa \alpha 1 \mu \varepsilon \lambda \varepsilon \dot{\tau} \tau$.

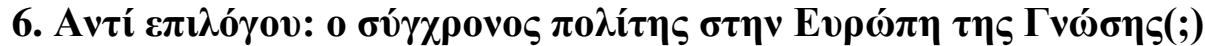

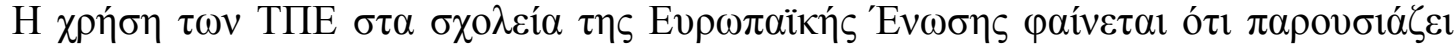

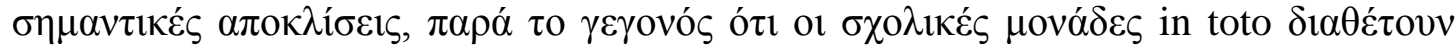

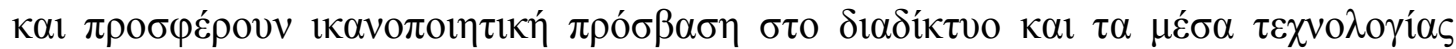

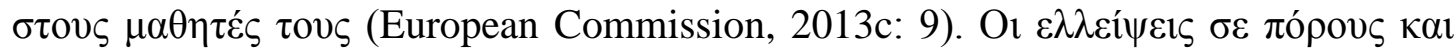

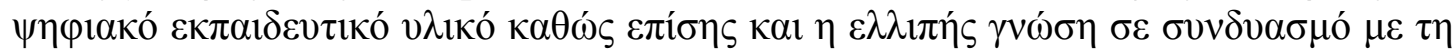

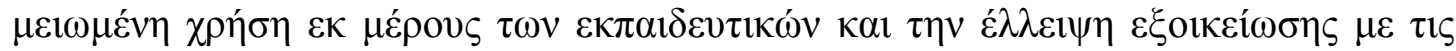

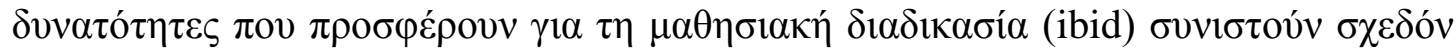

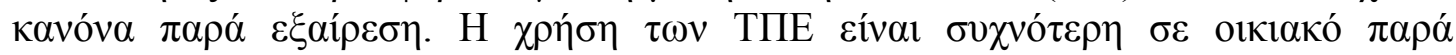

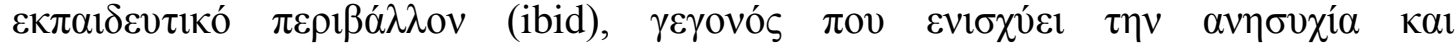

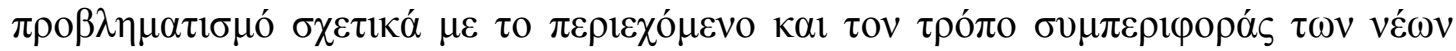

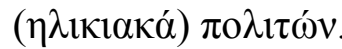




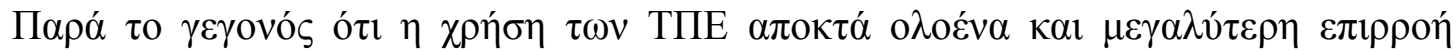

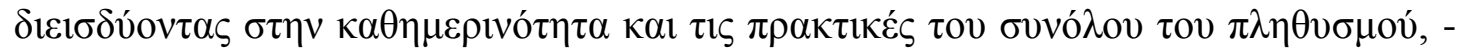

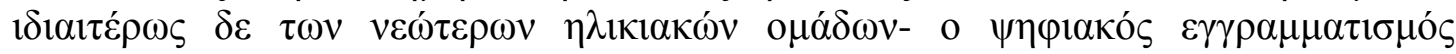

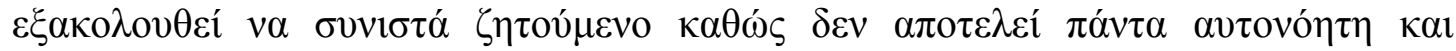

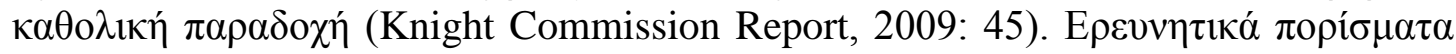

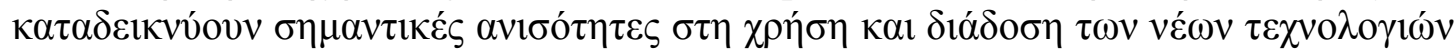

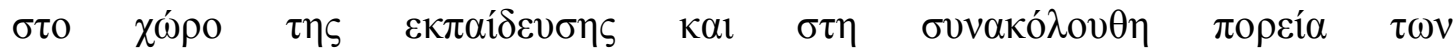

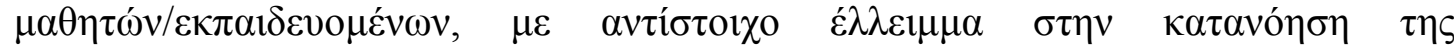

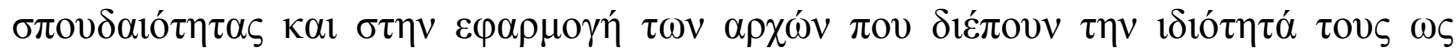

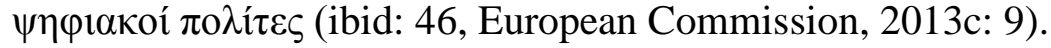

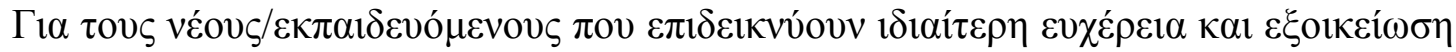

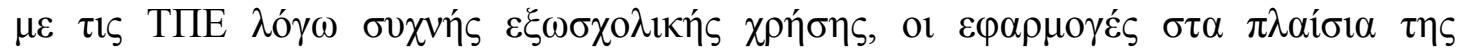

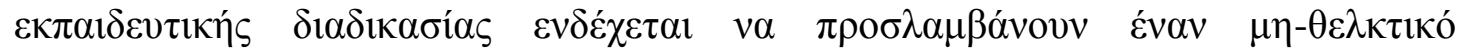

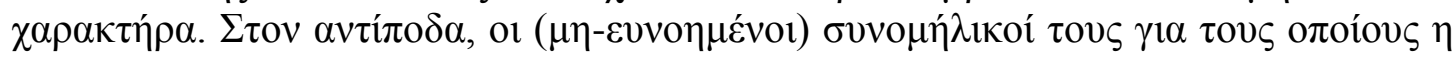

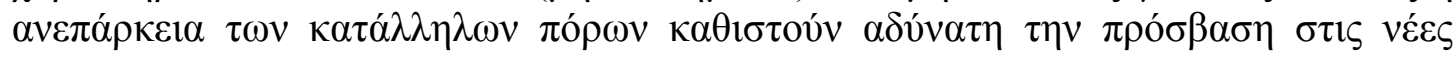

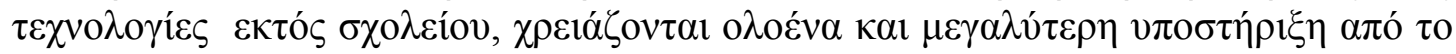

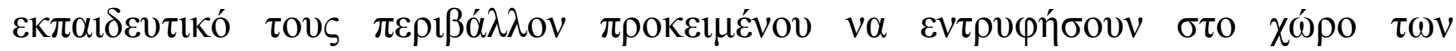

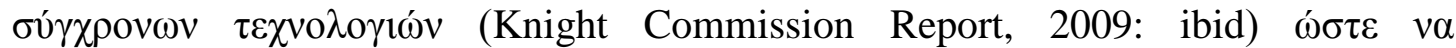

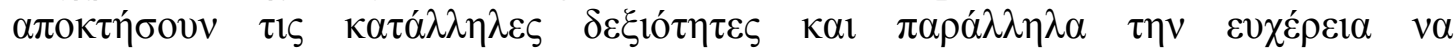

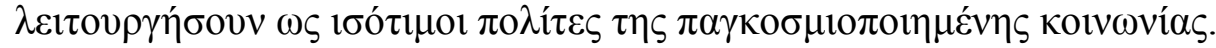

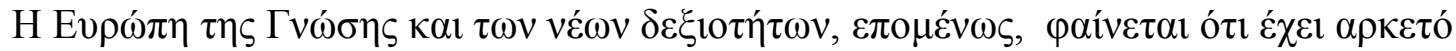

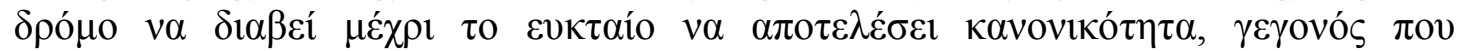

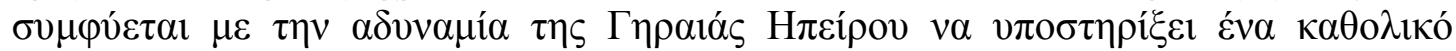

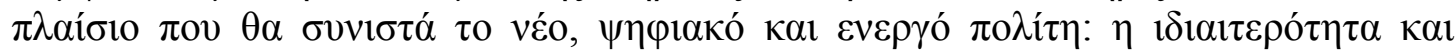

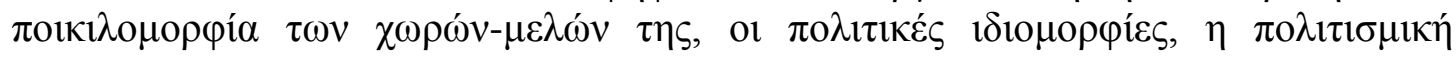

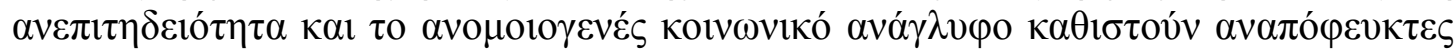

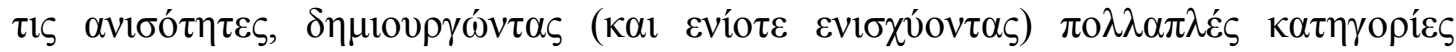

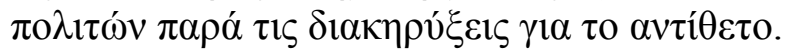

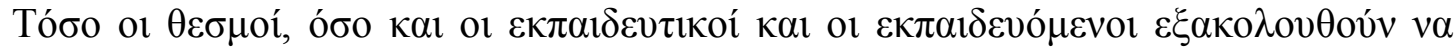

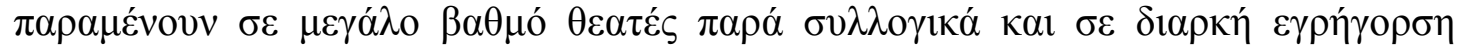

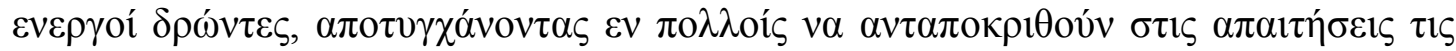

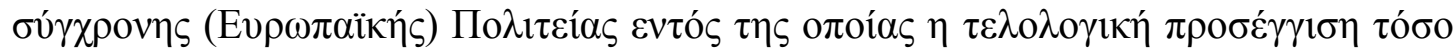

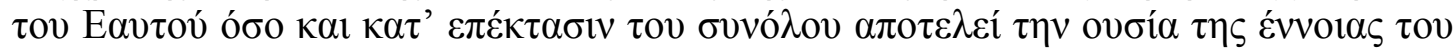

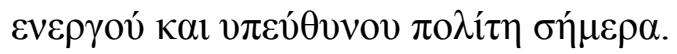

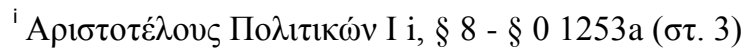

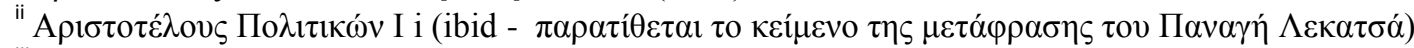

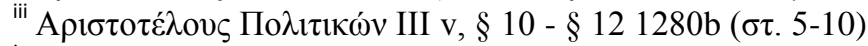

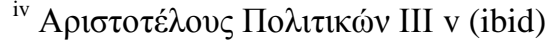

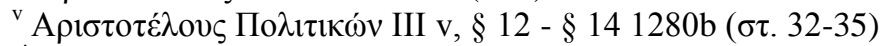

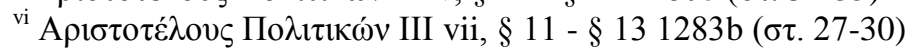




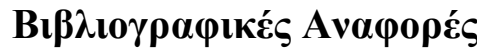

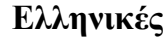

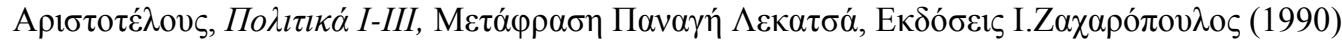

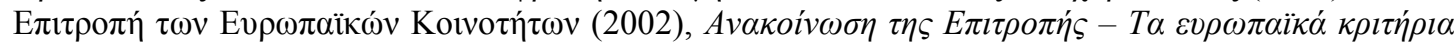

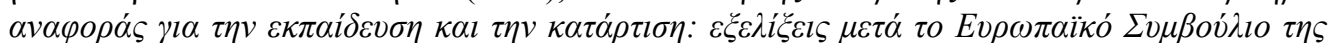

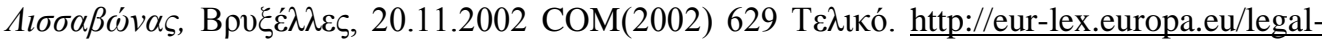
content/EL/TXT/PDF/?uri=CELEX:52002DC0629\&from=EL

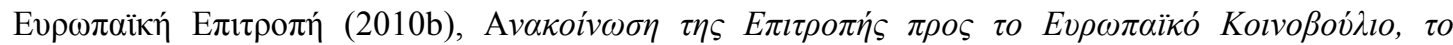

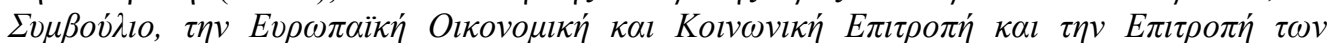

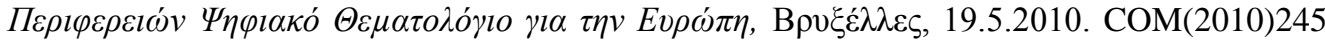

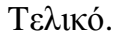
http://eur-lex.europa.eu/legal- content/EL/TXT/PDF/?uri=CELEX:52010DC0245\&from=EL

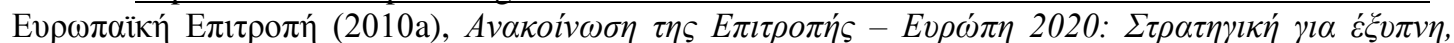

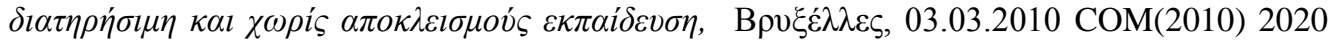

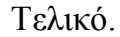

http://eur-lex.europa.eu/LexUriServ/LexUriServ.do?uri=COM:2010:2020:FIN:EL:PDF

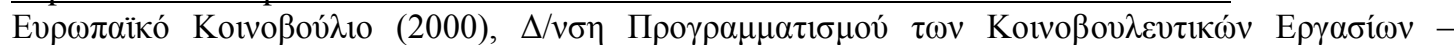

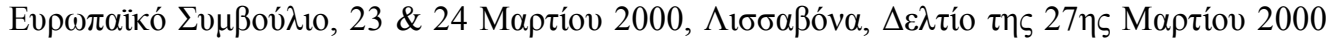
http://www.europarl.europa.eu/bulletins/pdf/1s2000el.pdf

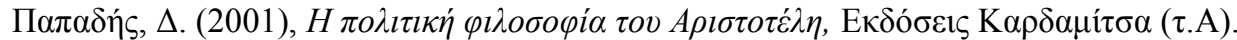

\section{$\Xi \varepsilon v o ́ \gamma \lambda \omega \sigma \sigma \varepsilon \varsigma$}

Anadiadou, K. \& Claro, M. (2009), $21^{\text {st }}$ Century Skills and Competences for New Millennium Learners in OECD Countries, OECD Working Papers, No. 41, OECD Publishing. http://dx.doi.org/10.1787/218525261154

Bacon, F. (1597), 'Meditationes Sacrae De Haeresibus' as quoted in The Concise Oxford Dictionary of Proverbs New Edition (2003), Simpson J. \& Speake J. (ed.), Oxford University Press

Bellamy, R., Castiglione, D. (2003), Legitimizing the Euro-'Polity' and its 'Regime' The Normative Turn in EU Studies, European Journal of Political Theory 2003; 2: pp. 7-34, DOI: $10.1177 / 1474885103002001277$

Castells, M. (2000), Materials for an exploratory theory of the network society, British Journal of Sociology Vol. No. 51 Issue 1 (January/March 2000) pp. 5-24 London School of Economics 2000

Commission of the European Communities (2005), Proposal for a Recommendation of the European Parliament and the Council on Key Competencies for Lifelong Learning (presented by the Commission), Brussels, 20.11.2005 COM(2005) 548 Final. 2005/0221(COD) http://www.europarl.europa.eu/meetdocs/2004_2009/documents/com/com_com\%282005\%29 0548 /com com\%282005\%290548 en.pdf

Commission of the European Communities (2002), Proposal for a DECISION OF THE EUROPEAN PARLIAMENT AND OF THE COUNCIL adopting a multi-annual programme (2004-2006) for the effective integration of Information and Communication Technologies (ICT) in education and training systems in Europe (eLearning Programme, Brussels, 19.12.2002, $\operatorname{COM}(2002) 751$ final, 2002/0303 (COD)

Deleuze, G., Guattari, F. (1987), A Thousand Plateaus', Minneapolis, University of Minnessota Press (tr. Brian Massumi)

https://www.ntnu.no/wiki/download/attachments/21463142/deleuzeguattarirhizome.pdf European Communities (2014), eSkills for Jobs in Europe, Measuring Progress and Moving Ahead, European Commission DG Enterprise and Industry Key Enabling Technologies and Digital Economy, Brussels, Ref. Ares(2014)1102611 - 08/04/2014.

European Commission (2013c), Survey of Schools: ICT in Education Benchmarking Access, Use and Attitudes to Technology in Europe's Schools, FINAL REPORT A study prepared for the European Commission DG Communications Networks, Content \& Technology, February 2013 https://ec.europa.eu/digital-agenda/sites/digital-agenda/files/KK-31-13-401-EN-N.pdf

European Commission (2013b), Communication from the Commission to the European Parliament, the Council, the European Economic and Social Committee and the Committee of the Regions Opening up Education: Innovative teaching and learning for all through new Technologies and Open Educational Resources \{SWD(2013) 341 final\}, Brussels, 25.9.2013 COM(2013) 654 final http://eur-lex.europa.eu/legalcontent/EN/TXT/PDF/?uri=CELEX:52013DC0654\&from=EN 
European Commission (2013a), DIGCOMP: A Framework for Developing and Understanding Digital Competence in Europe, European Commission Joint Research Centre Institute for Prospective Technological Studies, Luxembourg: Publications Office of the European Union, 2013 https://ec.europa.eu/jrc/sites/default/files/lb-na-26035-enn.pdf

Flora, K. (2014), Internet addiction disorder among adolescents and young adults: the picture in Europe and prevention strategies in Perspectives of Youth: Connections and disconnections, Volume 2, Partnership of the European Commission and the Council of Europe, Council of Europe Publishing : pp eu.coe.int/documents/1017981/8422373/Perspectives-on-youth-vol.+2.pdf/17ac5405-846e4dca-a326-983fc0f2ded5

Hobbs A., Jensen A. (2009), The Past, Present, and Future of Media Literacy Education, Journal of Media Literacy Education 1: pp. 1-11.

Jochun, V., Pratten, B. \& Wilding, K. (2005), Civil renewal and active citizenship, A Guide to the Debate, NVCO $\quad$ http://socialwelfare.bl.uk/subject-areas/government-issues/socialpolicy/ncvo/138025civil_renewal_active citizenship.pdf

Jones, E., Gaveta, J. (2002), Concepts of Citizenship: A Review, Institute of Development Studies, England https://www.ids.ac.uk/files/dmfile/Db19.pdf

Keeling, R. (2006), The Bologna Process and the Lisbon Research Agenda: the European Commission's expanding role in higher education discourse, European Journal of Education, Vol.41, No.2: 203-223

Keeting, A. (2009), Educating Europe's citizens: moving from national to post-national models of educating for European citizenship, Citizenship Studies Volume 13, Issue 2, 2009 Special Issue: Citizenship, Learning and Education; pp. 135-151 DOI:10.1080/13621020902731140,

Livingstone, S., Van Couvering, E., Thumin, N. (2008), Converging traditions of research on media and information literacies: disciplinary, critical, and methodological issues, LSE Research Online, The London School of Economics and Political Science http://eprints.lse.ac.uk/23564/

Millner, S. (2008), A Review of the Civil Renewal and Active Citizenship Debate, NVCO http://socialwelfare.bl.uk/subject-areas/government-issues/socialpolicy/ncvo/138028civil_renewal_active_citizenship_debate.pdf

Mossberger, K., Tolbert, C. J., McNeal, R. S. (2007), Digital Citizenship The Internet, Society, and Participation, MIT Press Books

Ólafsson, K., Livingstone, S., Haddon, L. (2014), Children's Use of Online Technologies in Europe. A review of the European evidence base, EU Kids Online network September 2014 (Revised Edition), The London School of Economics and Political Science, co-founded by the European

Union http://eprints.lse.ac.uk/60221/1/EU_Kids_Online_Children\%E2\%80\%99sUseofOnlineTechno logiesinEurope ReviewofEuropeanEvidenceBase_Revised_2014.pdf

Ribble, M. (2011), Digital Citizenship in Schools, International Society for Technology in Education (ISTE), $2^{\text {nd }}$ edition ISBN 978-1-56484-301-2

Ribble, M., Baily, R., Ross, T. (2004), Digital Citizenship, Addressing Appropriate Technology Behavior - ISTE, Learning \& Leading with Technology, September 2004

Simsek, E., Simsek, A. (2013), New Literacies for Digital Citizenship, Contemporary Educational Technology, 2013, 4(2), 126-137

Staksrud, E., Livingstone, S., Haddon, L., \& Ólafsson, K. (2009), What Do We Know About Children's Use of Online Technologies? A Report on Data Availability and Research Gaps in Europe (2nd edition), London School of Economics and Political Science, London: EU Kids Online. (EC Safer Internet plus Programme Deliverable D1.1) ISBN 978-0-85328-405-5 http://eprints.lse.ac.uk/24367/1/What\%20do\%20we\%20know\%20about\%20children\%E2\%80 $\% 99 \mathrm{~s} \% 20$ use \%20of\%20online\%20technologies\%281sero\%29.pdf

OECD (2015b), Skills Outlook 2015, OECD Publishing.

OECD (2015a), 'Education Policy Outlook 2015: Making Reforms Happen', OECD Publishing. http://dx.doi.org/10.1787/9789264225442-en

OECD (2014), Trends Shaping Education Spotlight 2014, OECD Publishing http://www.oecd.org/edu/ceri/Spotlight\%205-\%20Infinite\%20Connections.pdf

OECD (2012b), The Protection of Children Online: Recommendation of the OECD Council Report on risks faced by children online and policies to protect them, OECD Publishing http://www.oecd.org/sti/ieconomy/childrenonline_with_cover.pdf

OECD (2012a), Better Skills, Better Jobs, Better Lives: A Strategic Approach to Skills Policies, OECD Publishing. http://dx.doi.org/10.1787/9789264177338-en 
UNESCO, (2015), Global Citizenship Education, Topics and Learning Objectives, Paris: UNESCO.

UNESCO (2002), Technologies for Education, Potentials, Parameters and Prospects, UNESCO and the Academy for Educational Development.

World Bank Group (2011), Learning for All, Investing in People's Knowledge and Skills to Promote Development - Education Strategy $2020 \quad$ Executive Summary. http://siteresources.worldbank.org/EDUCATION/Resources/ESSU/Education_Strategy_4_12 2011.pdf

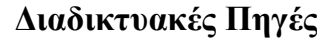

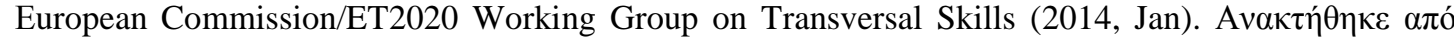
http://ec.europa.eu/education/policy/strategic-framework/expert-groups/documents/wgtransversal-mandate en.pdf ( $\tau \varepsilon \lambda \varepsilon v \tau \alpha i ́ \alpha \pi \rho \circ \sigma \varepsilon \dot{\lambda} \lambda \alpha \sigma \eta$ 05/09/2015)

Knight Commission Report (2009), Knight Commission on the Information Needs of Communities in a Democracy: Sustaining democracy in the digital age. Washinghton, DC, the Aspen Institute

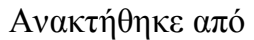

http://www.knightfoundation.org/media/uploads/publication_pdfs/Knight_Commission_Report_-

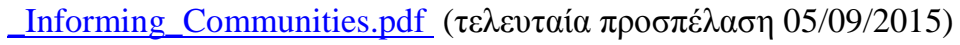

